\title{
The Influence of Gravity Waves on the Slope of the Kinetic Energy Spectrum in Simulations of Idealized Midlatitude Cyclones
}

\author{
Maximo Q. Menchaca and Dale R. DurRan \\ Department of Atmospheric Sciences, University of Washington, Seattle, Washington
}

(Manuscript received 5 November 2018, in final form 28 April 2019)

\begin{abstract}
The influence of gravity waves generated by surface stress and by topography on the atmospheric kinetic energy (KE) spectrum is examined using idealized simulations of a cyclone growing in baroclinically unstable shear flow. Even in the absence of topography, surface stress greatly enhances the generation of gravity waves in the vicinity of the cold front, and vertical energy fluxes associated with these waves produce a pronounced shallowing of the KE spectrum at mesoscale wavelengths relative to the corresponding free-slip case. The impact of a single isolated ridge is, however, much more pronounced than that of surface stress. When the mountain waves are well developed, they produce a wavenumber to the $-5 / 3$ spectrum in the lower stratosphere over a broad range of mesoscale wavelengths. In the midtroposphere, a smaller range of wavelengths also exhibits a $-5 / 3$ spectrum. When the mountain is $500 \mathrm{~m}$ high, the waves do not break, and their KE is entirely associated with the divergent component of the velocity field, which is almost constant with height. When the mountain is $2 \mathrm{~km}$ high, wave breaking creates potential vorticity, and the rotational component of the KE spectrum is also strongly energized by the waves. Analysis of the spectral KE budgets shows that the actual spectrum is the result of continually shifting balances of direct forcing from vertical energy flux divergence, conservative advective transport, and buoyancy flux. Nevertheless, there is one interesting example where the $-5 / 3$-sloped lower-stratospheric energy spectrum appears to be associated with a gravity-waveinduced upscale inertial cascade.
\end{abstract}

\section{Introduction}

The seminal analysis of data collected on commercial aircraft by Nastrom and Gage (1985) established that, on average, the midlatitude horizontal kinetic energy (KE) spectrum in the upper troposphere and lower stratosphere follows a $k^{-3}$ slope at large scales with a gradual transition at wavelengths around $400 \mathrm{~km}$ to a $k^{-5 / 3}$ slope at smaller scales, where $k$ is the horizontal wavenumber. The $k^{-3}$ portion of the spectrum is widely accepted as arising from an enstrophy cascade from large to small scales in approximate agreement with two-dimensional turbulence theory (Kraichnan 1967; Lindborg 1999) or quasigeostrophic turbulence (Charney 1971). In contrast, there is much less agreement on the processes responsible for the $k^{-5 / 3}$ slope in the mesoscale, which encompasses horizontal scales between roughly 4 and $400 \mathrm{~km}$.

As shown by Kolmogorov and Obukhov (Vallis 2017, p. 442) a $k^{-5 / 3}$ spectral slope can be produced by an

Corresponding author: Dale R. Durran,drdee@uw.edu inertial cascade of energy, and in three-dimensional homogeneous isotropic turbulence, this cascade is from large to small scales. But in the atmosphere, the timemean horizontal KE spectrum exhibits a $k^{-5 / 3}$ slope at scales far too large to be characterized by homogeneous isotropic motions. Several authors have therefore proposed alternative mechanisms through which horizontal KE might cascade through the mesoscale, including from large to small scales via gravity waves (Dewan 1979; VanZandt 1982), or from small to large scales via stratified turbulence developing in regions such as thunderstorm anvils (Gage 1979; Lilly 1983).

Nevertheless, the $k^{-5 / 3}$ spectral slope does not have to be produced through an inertial cascade, and recent research supports the idea that energy injected into the small scales by gravity waves and deep convection might directly force the spectrum. Waite and Snyder (2009) simulated cyclones developing in a baroclinically unstable channel flow using $25-\mathrm{km}$ horizontal grid spacing and found that gravity waves radiating from regions of geostrophic imbalance created a $k^{-5 / 3}$ slope over the short-wavelength end of the spectrum in the lower 
stratosphere. The spectral energy budget for their simulations showed the stratospheric KE distribution was not produced solely by an inertial cascade, but also by direct forcing from vertical pressure flux divergence over much of the mesoscale. The simulations in Waite and Snyder (2009) were dry and underenergized compared to observations. When these simulations were extended to include moisture and parameterized deep convection, gravity wave activity was enhanced and transitions from $k^{-3}$ to $k^{-5 / 3}$ spectral slopes developed in both the troposphere and the stratosphere (Waite and Snyder 2013). As in the dry case, the $k^{-5 / 3}$ spectral slope was not produced exclusively by an inertial cascade; rather the spectral energy budget showed contributions to the horizontal KE from buoyancy forces over a broad range of scales. Peng et al. (2015a) provided a more detailed analysis of the spectral energy budget for an idealized developing cyclone in a moist baroclinically unstable channel flow similar to that considered in Waite and Snyder (2013). Again using a horizontal grid spacing of $25 \mathrm{~km}$, they found that direct forcing, from vertical pressure fluxes and horizontal KE fluxes, provided important contributions to the lower stratosphere $\mathrm{KE}$ spectrum over a broad range of mesoscale wavelengths. Augier and Lindborg (2013) examined 10 days of global simulations with the Atmospheric General Circulation Model for the Earth Simulator (AFES) run at $60-\mathrm{km}$ horizontal resolution (T639) and 25 days of global simulations with the European Centre for Medium-Range Weather Forecasts (ECMWF) Integrated Forecast System (IFS) at roughly 30-km resolution (T1279). In both datasets they found that the stratospheric mesoscales were directly forced by upward energy fluxes associated with gravity waves propagating up from the troposphere, although in the AFES simulations, the vertical energy flux divergence was less important than a strong downscale energy cascade.

Using convection-permitting horizontal grid spacings of $1 \mathrm{~km}$, Durran and Weyn (2016) showed a $k^{-5 / 3}$ horizontal KE spectrum can be generated in an initially quiescent horizontally uniform environment by idealized convective systems. Sun et al. (2017) analyzed the spectral KE budget in simulations similar to those in Durran and Weyn (2016) and showed that buoyancy forces and vertical energy fluxes play an important role in regulating the $\mathrm{KE}$ spectrum across a wide range of scales, again suggesting the $k^{-5 / 3}$ slope is not exclusively generated through an inertial cascade.

Mountains have long been identified as both a source of internal gravity waves and as regions in which observations show enhanced horizontal velocity variance (Nastrom and Gage 1985; Nastrom et al. 1987; Jasperson et al. 1990; Nastrom and Fritts 1992). Numerical simulations of the global atmospheric circulation can reproduce the transition between the $k^{-3}$ and $k^{-5 / 3}$ slopes in the horizontal KE spectrum (Koshyk and Hamilton 2001; Hamilton et al. 2014; Skamarock et al. 2014). Malardel and Wedi (2016) used a dry adiabatic version (no surface friction, no surface heat fluxes) of one such model to investigate the influence of orography on the horizontal $\mathrm{KE}$ spectrum and found Earth's topographic forcing greatly enhanced the energy in the mesoscale portion of the spectrum, shallowing the spectral slope from $k^{-3}$ to something only slightly steeper than $k^{-5 / 3}$.

In this paper we again consider a dry baroclinically unstable channel flow, similar to that in Waite and Snyder (2009), to investigate the influence of a single isolated ridge on the domain-averaged horizontal KE spectrum. Our previous research on mountain waves generated in such flows (Menchaca and Durran 2017, hereafter MD17) showed that in the absence of surface friction, the surface winds around the developing cyclone become too strong to produce realistic mountain waves. Further evidence for the potential importance of surface friction was obtained by Malardel and Wedi (2016), who found that the horizontal KE on scales shorter than about $400 \mathrm{~km}$ in their free-slip dry adiabatic model with terrain exceeded the $\mathrm{KE}$ in a full-complexity reference simulation that included moisture and the full suite of standard convective and other physical parameterizations. We therefore include a simple parameterization of boundary layer stress in all of our simulations, except for a no-drag, nomountain reference case similar to that in Waite and Snyder (2009).

In the following, section 2 provides a brief overview of the numerical model and the simulations. The horizontal and vertical KE spectra for the suite of simulations are presented in section 3. Section 4 gives an analysis of the spectral energy budgets. Section 5 contains the conclusions.

\section{Numerical model and overview of the simulations}

The large-scale flow and the initiation of the cyclone are described in MD17, along with the shape of the isolated ridge, whose approximate $x$ and $y$ extents are 80 and $640 \mathrm{~km}$. In addition to simulations with no topography, the same two mountain heights are again considered: $500 \mathrm{~m}$ and $2 \mathrm{~km}$. All simulations are conducted with the Advanced Research version of the Weather Research and Forecasting Model (WRF-ARW) using a horizontal domain $L_{x}=16200 \mathrm{~km}$ wide and periodic along the east-west $(x)$ axis with symmetric side walls bounding its $L_{y}=9000-\mathrm{km}$ north-south $(y)$ extent. The domain is $20.5 \mathrm{~km}$ deep with the top $6 \mathrm{~km}$ devoted to a 


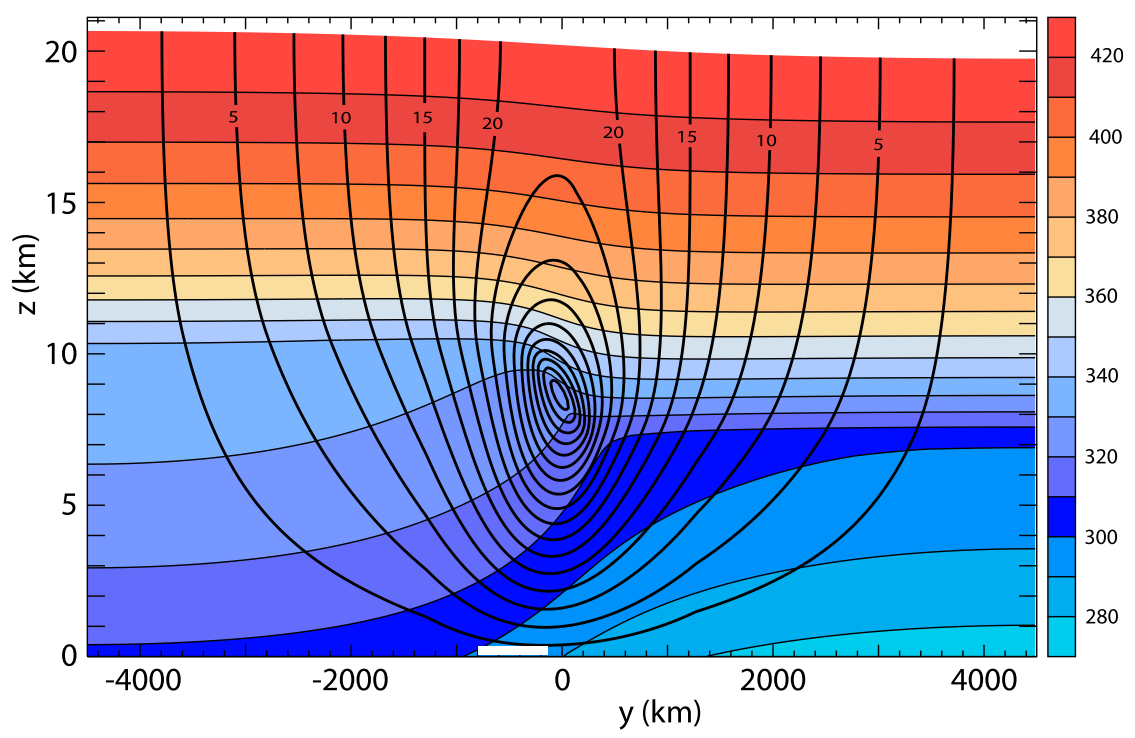

FIG. 1. North-south cross section of background shear flow: $\theta$ (colors, $10-\mathrm{K}$ intervals) and zonal velocity (black, $2.5 \mathrm{~m} \mathrm{~s}^{-1}$ intervals). The white bar extending from $-800 \leq y \leq-160 \mathrm{~km}$ shows the north-south extent of the ridge. Data are not plotted in the wave-absorbing layer.

Rayleigh-damping layer tuned to absorb vertically propagating gravity waves (as detailed in MD17). The horizontal grid spacing is $\Delta x=\Delta y=12 \mathrm{~km}$; there are 80 vertical levels spaced at $30 \mathrm{~m}$ near the surface, with $\Delta z$ increasing to roughly $240 \mathrm{~m}$ around the tropopause and $400 \mathrm{~m}$ near the model top. The time step is $40 \mathrm{~s}$. The only physical parameterizations we employ are 2D Smagorinsky mixing in the horizontal and a modified version of the Yonsei University (YSU) boundary layer scheme (Hong et al. 2006) to limit the winds around the cyclone to realistic values though surface friction and vertical mixing. Default values of the parameters are used for the boundary layer scheme except that the heat flux is set to zero, $z_{0}$ is a uniform $0.1 \mathrm{~m}$, and the boundary layer height is fixed at the sixth model level, approximately $282 \mathrm{~m}$ above the surface.

Figure 1 is a $y-z$ cross section showing isotachs of the westerly wind component and isentropes of potential temperature in the baroclinically unstable shear flow. The north-south position of the mountain is shown by the white bar above the $y$ axis, which is just south of the core of the jet. As discussed in MD17, a cyclone is triggered by an isolated PV perturbation; its evolution between 3.5 and 6.5 days is illustrated by the surface isobars and surface potential temperature fields plotted in Fig. 2 for the simulation with flat terrain. The position occupied by the mountain, in those simulations in which it is present, is indicated in Fig. 2 by the black vertical bar centered at $(x, y)=(0,-480) \mathrm{km}$. Note that the mountain covers roughly $0.1 \%$ of the surface area containing significant surface pressure perturbations at 6.5 days.
In the simulations with topography, mountain waves are weak at 3.5 days, when the cyclone is well upstream of the terrain (Fig. 2a). As the cold front passes the mountain at 4.5 days, waves amplify (Fig. 2b), and strong transient wave activity persists past 5.5 days (Fig. 2c), before slackening toward the end of the simulation as the cyclone propagates zonally past the mountain (Fig. 2d).

Throughout the following, we focus on four simulations; three use the YSU boundary layer scheme and terrain heights of 0,500 , and $2000 \mathrm{~m}$ and are denoted $\mathrm{H} 0$, $\mathrm{H} 500$, and $\mathrm{H} 2000$, respectively. The fourth simulation, HOFS, has flat terrain and a free-slip lower boundary. As shown by the comparison of the vertical velocity fields in Fig. 3, at 5.5 days many more short-wavelength gravity waves are generated in $\mathrm{H} 0$ than in the corresponding free-slip simulation H0FS. These waves originate along the cold front and in the cold air mass southeast of the low center and propagate upward into the stratosphere, where they are quite prominent at $z=12 \mathrm{~km}$ (Fig. 3d), although their vertical-velocity amplitudes are not high $\left[O(1) \mathrm{cm} \mathrm{s}^{-1}\right]$.

Several previous investigations of gravity waves generated during free-slip simulations of the life cycle of baroclinic waves have found a broad spectrum of gravity waves generated through a variety of mechanisms in different sectors around the cyclone (e.g., Plougonven and Snyder 2007; Kim et al. 2016). In comparison to these previous free-slip simulations, the gravity wave packets appearing in the surface-stress simulation $\mathrm{H} 0$ are unusually monochromatic and well defined, with an average wavelength of about $70 \mathrm{~km}$, or roughly $6 \Delta x$. 

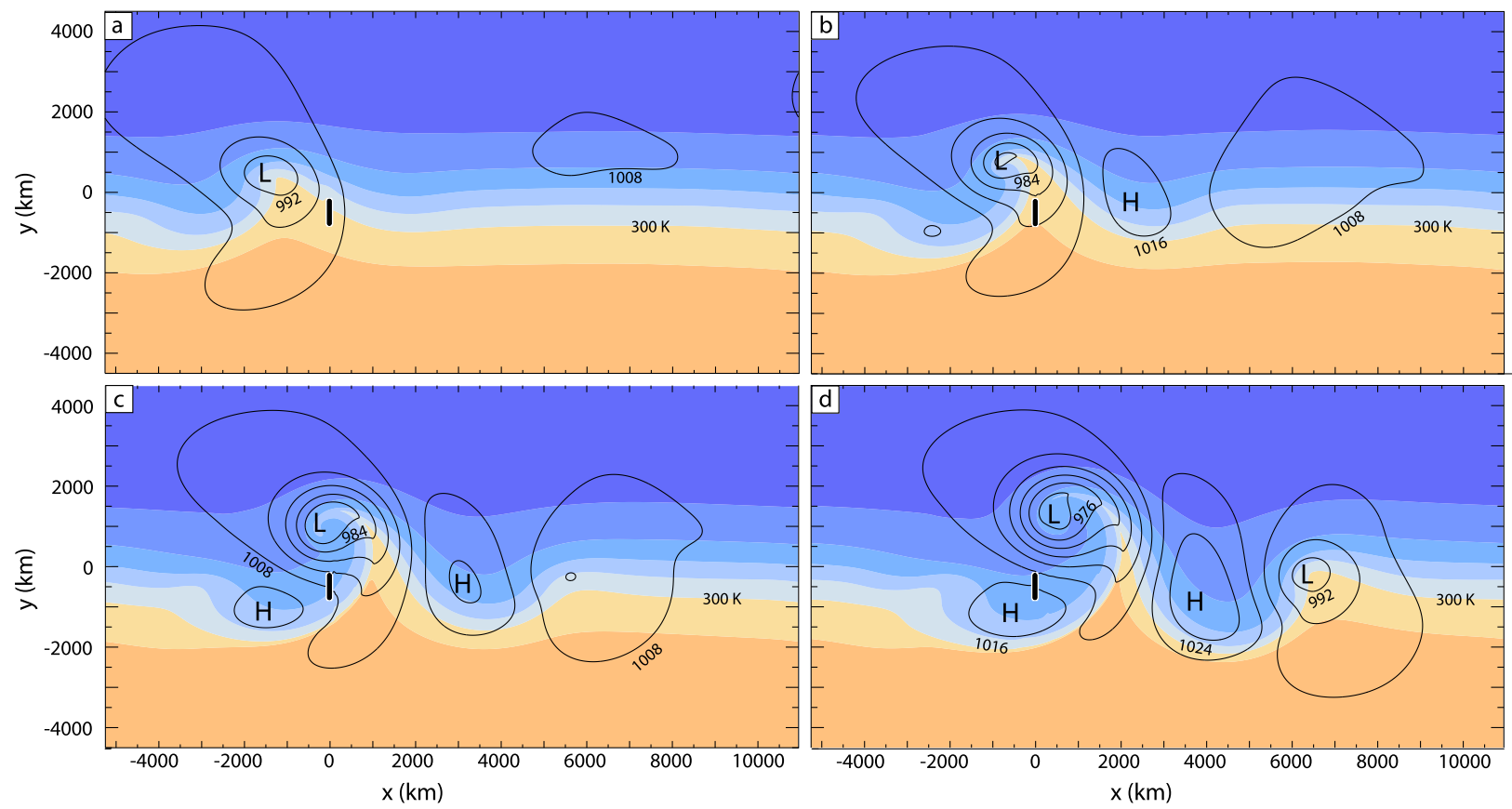

FIG. 2. Surface isobars (black, 8-hPa intervals) and surface $\theta$ (color, 5-K intervals, 300-K isoline is labeled) for the developing cyclone in the no-mountain simulation at (a) 3.5, (b) 4.5, (c) 5.5, and (d) 6.5 days. The position occupied by the mountain in simulations where it is present is depicted by the black vertical bar at $x=0 \mathrm{~km}$. Lows and highs are labeled by " $\mathrm{L}$ " and " $\mathrm{H}$," respectively.

As such the waves are poorly resolved, and, in light of the findings of Snyder et al. (1993) about spurious gravity waves appearing in studies of $2 \mathrm{D}$ frontogenesis, it is possible they are a numerical artifact. To test this, the simulation was repeated with a nested mesh having a horizontal grid spacing of $4 \mathrm{~km}$. As shown in Fig. 4 the vertical velocities in the waves on the finer mesh are stronger by roughly a factor of 3 , and shorter wavelengths appear. Nevertheless, the reduction in the wavelength in the region cut by the green line segment is modest, and it is nowhere near as great as the factor-of-3 decrease in the grid spacing. The average wavelength along the $340-\mathrm{km}$-long line segment is $68 \mathrm{~km}$ in the $12-\mathrm{km}$-mesh simulation and $52 \mathrm{~km}$ in the $4-\mathrm{km}$ simulation. The wavelength in the $4-\mathrm{km}$ simulation is, therefore, roughly $13 \Delta x$, which is adequate numerical resolution. Closer to the surface, at $z=1 \mathrm{~km}$, the wellorganized wave pattern in Fig. 3 becomes a more complex superposition of waves on the $4-\mathrm{km}$ mesh. A thorough investigation of the generation and propagation of these waves is left for future study.

\section{Spectra}

Energy spectra are obtained by first linearly interpolating the WRF data to constant- $z$ surfaces at vertical intervals of $250 \mathrm{~m}$. The fields, which are already periodic in $x$, are made doubly periodic using the rigid-wall boundary conditions to extend the data in $y$ : the meridional velocity $v$ is odd-extended, while the remaining variables are even-extended (Waite and Snyder 2009). The resulting data, which now fill a domain of size $L_{x}$ by $2 L_{y}$ are Fourier transformed from $(x, y)$ physical space to wavenumber space, where $\mathbf{k}=k_{x} \mathbf{i}+k_{y} \mathbf{j}$ is the wavenumber vector. The horizontal and vertical KE spectra are calculated as

$$
\begin{aligned}
& E_{h}(\mathbf{k}) \equiv \frac{1}{2} \bar{\rho}\left[\hat{u}^{*}(\mathbf{k}) \hat{u}(\mathbf{k})+\hat{v}^{*}(\mathbf{k}) \hat{v}(\mathbf{k})\right]=\frac{1}{2} \bar{\rho} \hat{\mathbf{u}}^{*}(\mathbf{k}) \cdot \hat{\mathbf{u}}(\mathbf{k}), \\
& E_{v}(\mathbf{k}) \equiv \frac{1}{2} \bar{\rho} \hat{w}^{*}(\mathbf{k}) \hat{w}(\mathbf{k})
\end{aligned}
$$

where $\mathbf{u}=(u, v)$ is the horizontal velocity vector, $w$ is the vertical velocity, Fourier-transformed variables are indicated by hats, complex conjugates are denote by the star superscript, and $\bar{\rho}(z)$ is a horizontally uniform background density profile. These $2 \mathrm{D}$ spectra are binned by the magnitude of the horizontal wavenumber $k=|\mathbf{k}|$, with bin size $\Delta k=2 \pi / L_{y}$, such that for an arbitrary field $a$,

$$
\hat{a}(k)=\sum_{\mathbf{k} \in R(k)} \hat{a}(\mathbf{k})
$$


Free-Slip
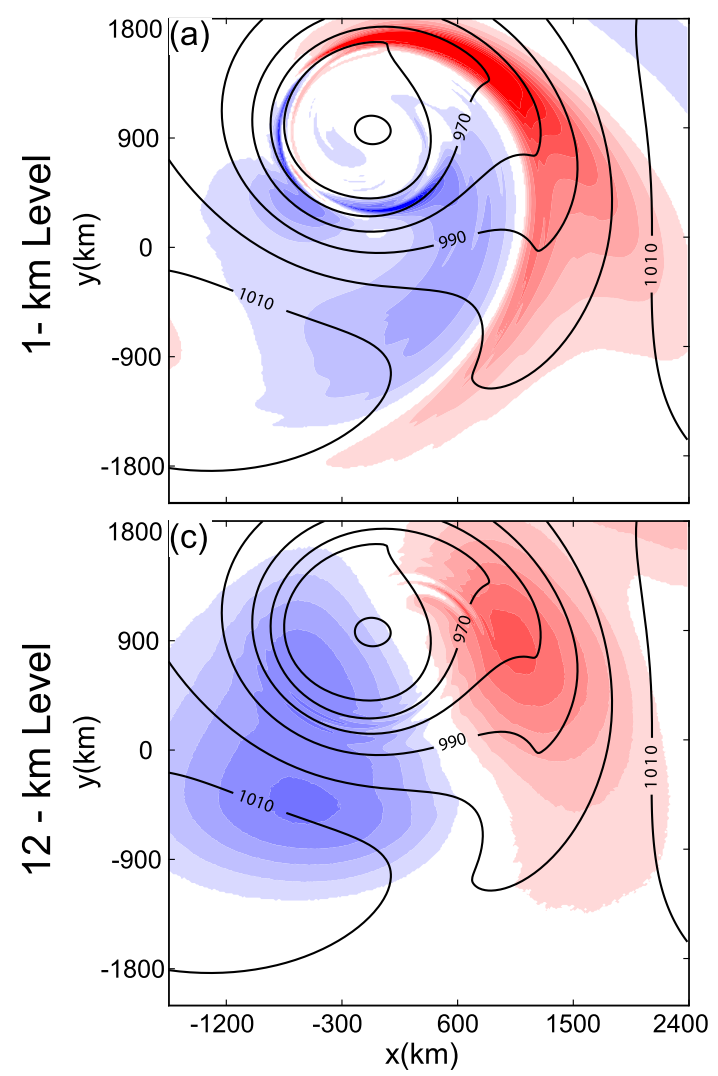

Surface Stress


FIG. 3. The vertical velocity $w$ (color, $\mathrm{m} \mathrm{s}^{-1}$ ) at 5.5 days at (a),(b) $z=1$ and (c),(d) $z=12 \mathrm{~km}$ from the (a),(c) free-slip simulation (H0FS) and (b),(d) surface stress simulation (H0). Also plotted for reference are isobars on the $50 \mathrm{~m}$ AGL surface, contoured every $10 \mathrm{hPa}$.

where $R(k)$ is the set of $\mathbf{k}$ satisfying

$$
k-\frac{\Delta k}{2} \leq|\mathbf{k}|<k+\frac{\Delta k}{2} .
$$

Data are plotted as a function of the nondimensional discrete horizontal wavenumber $\tilde{k} \equiv\left(L_{y} / 2 \pi\right) k$, and smoothed to reduce the noise introduced by $2 \mathrm{D}$ binning using the technique in Durran et al. (2017).

\section{a. Horizontal KE spectra}

Spectra of the kinetic energy of the horizontal wind $E_{h}(\tilde{k})$ for all four simulations are plotted in Fig. 5. Spectra from the midtroposphere, vertically averaged between 3.5 and $5.5 \mathrm{~km}$, appear in the left column; spectra from the lower stratosphere, averaged between 11.5 and $13.5 \mathrm{~km}$ are in the right column. The $11.5-13.5-\mathrm{km}$ layer is within the 9-14-km range over which data collected by commercial aircraft showed that, on average, the slopes of the atmospheric horizontal kinetic energy spectrum are $k^{-3}$ and $k^{-5 / 3}$ on the large-scale and mesoscale, respectively (Nastrom and Gage 1985). Reference lines with slopes of $\tilde{k}^{-3}$ and $\tilde{k}^{-5 / 3}$ are therefore also plotted in each panel.

We first examine the influence of surface stress by comparing the two no-mountain simulations, whose spectra are plotted as solid (H0) and dashed (H0FS) red curves. At both 4.5 (Fig. 5a) and 6.5 days (Fig. 5c), the lower-tropospheric spectrum for $\mathrm{H} 0$ clearly has a flatter slope than that for H0FS for mesoscale wavelengths $\lambda$ between roughly 50 and $110 \mathrm{~km}$. By day 6.5 , the slope in this wavelength band for $\mathrm{H} 0$ has become very close to $\tilde{k}^{-5 / 3}$, while that for HOFS is much steeper than $\tilde{k}^{-3}$. On the larger synoptic scale, for wavelengths between 450 and $4500 \mathrm{~km}$, the spectral slopes for both $\mathrm{H} 0$ and H0FS are close to $\tilde{k}^{-3}$ by day 6.5 . The situation is qualitatively similar in the lower stratosphere (Figs. 5b,d), although at this higher level, the synoptic-scale slopes for both simulations remain steeper than $\tilde{k}^{-3}$, while the mesoscale slopes for $\mathrm{H} 0$ are flatter than $\tilde{k}^{-5 / 3}$. The mesoscale slopes for the free-slip case H0FS are significantly shallower than those at the corresponding times in the midtroposphere, but still steeper that the slopes for $\mathrm{H} 0$. 

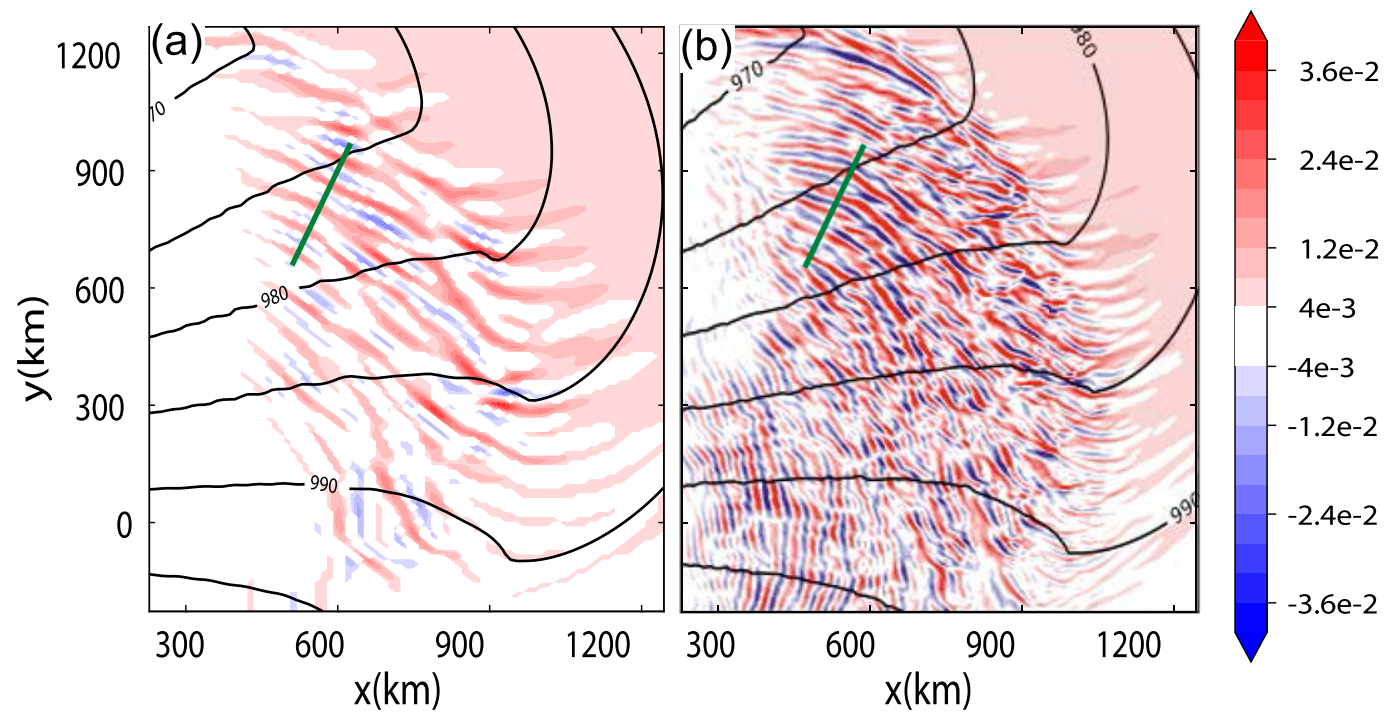

FIG. 4. The vertical velocity $w$ (color, $\mathrm{m} \mathrm{s}^{-1}$ ) at $z=12 \mathrm{~km}$ in (a) a zoom of Fig. $3 \mathrm{~d}$ into the region southeast of the cyclone center and (b) the same region from a nested simulation of the same case with 4-km horizontal grid spacing. Also plotted for reference are isobars on the $50 \mathrm{~m}$ AGL surface, contoured every $10 \mathrm{hPa}$ and a $340-\mathrm{km}-$ long reference line roughly perpendicular to the wave fronts (green).

The spectral slopes steepen due to numerical dissipation


be discussed later in more detail, the mesoscale shallowing of the spectrum of H0 relative to H0FS is associated with short-wavelength gravity waves generated near the surface that are not present in HOFS, as apparent in the vertical velocity fields in Figs. 3 and 4.

Much stronger gravity waves are generated in the simulations with the mountain ridge, and as evident in Fig. 5, there is substantially more $E_{h}(\tilde{k})$ at mesoscale wavelengths in these simulations and a broader range of wavelengths over which the spectral slopes are flatter than in the H0 simulation. At 4.5 days in the midtroposphere (Fig. 5a), both H500 (green) and H2000 (blue) are clearly more energetic at scales below $\lambda=300 \mathrm{~km}$, with the spectral slopes equal to or shallower than $\tilde{k}^{-5 / 3}$ (Fig. 5a). By day 6.5 (Fig. 5c), the H2000 simulation shows increased values of $E_{h}(\tilde{k})$ beyond those in $\mathrm{H} 0$ for all wavelengths shorter than $750 \mathrm{~km}$. Similarly, the H500 simulation has increased values of $E_{h}(\tilde{k})$ relative to $\mathrm{H} 0$ for all wavelengths shorter than $250 \mathrm{~km}$. An approximate $\tilde{k}^{-5 / 3}$ slope is seen in $E_{h}$ for the $\mathrm{H} 500$ case between wavelengths of 90 and $180 \mathrm{~km}$, whereas the $\mathrm{H} 2000$ simulation exhibits a roughly $\tilde{k}^{-3}$ slope between wavelengths of $900 \mathrm{~km}$ and the dissipation scale.

The influence the mountain is even more dramatic in the lower stratosphere (Figs. 5b,d). Unlike in the troposphere, the spectrum of $E_{h}$ decays more rapidly than $k^{-3}$ on the synoptic scales. Just slightly before the cold front arrives at the mountain, flattened, and even positive, spectral slopes are present on the mesoscale for both $\mathrm{H} 500$ and $\mathrm{H} 2000$ (Fig. 5b) and $E_{h}$ is enhanced relative to the $\mathrm{H} 0$ case through longer wavelengths than those in the troposphere. The horizontal KE spectra exhibit relative minima around wavelengths of $360 \mathrm{~km}$ for $\mathrm{H} 500$ and $180 \mathrm{~km}$ for $\mathrm{H} 2000$, suggesting that at this early time, the terrain's influence is strongest at small scales. By 6.5 days, the spectra for both $\mathrm{H} 500$ and $\mathrm{H} 2000$ exhibit clear $\tilde{k}^{-5 / 3}$ slopes between wavelengths of roughly $1000 \mathrm{~km}$ and the dissipation scale, and the $E_{h}$ values for the 2-km-high-mountain case are about an order of magnitude higher than those for the 500-m-mountain case. These results suggest that even isolated mountains of modest height can at least temporarily generate the observed $k^{-5 / 3}$ slope of the mesoscale kinetic energy spectrum.

\section{b. Rotational and divergent components of the horizontal KE spectrum}

The horizontal kinetic energy at wavenumber $\mathbf{k}$ is the sum of the rotational $E_{r}(\mathbf{k})$ and divergent $E_{d}(\mathbf{k})$ components of the horizontal wind, ${ }^{1}$

$$
E_{r}(\mathbf{k})=\frac{1}{2} \bar{\rho} \frac{\left.\hat{\zeta}(\mathbf{k})\right|^{2}}{k^{2}} \text { and } \quad E_{d}(\mathbf{k})=\frac{1}{2} \bar{\rho} \frac{|\hat{\delta}(\mathbf{k})|^{2}}{k^{2}}
$$

where $\zeta=\partial_{x} v-\partial_{y} u$ is the vertical component of the vorticity, and $\delta=\partial_{x} u+\partial_{y} v$ is the horizontal divergence.

\footnotetext{
${ }^{1}$ Because our domain is periodic in $x$ and has symmetric northern and southern boundaries, there is no nontrivial contribution from a nondivergent irrotational velocity field.
} 
Mid Troposphere

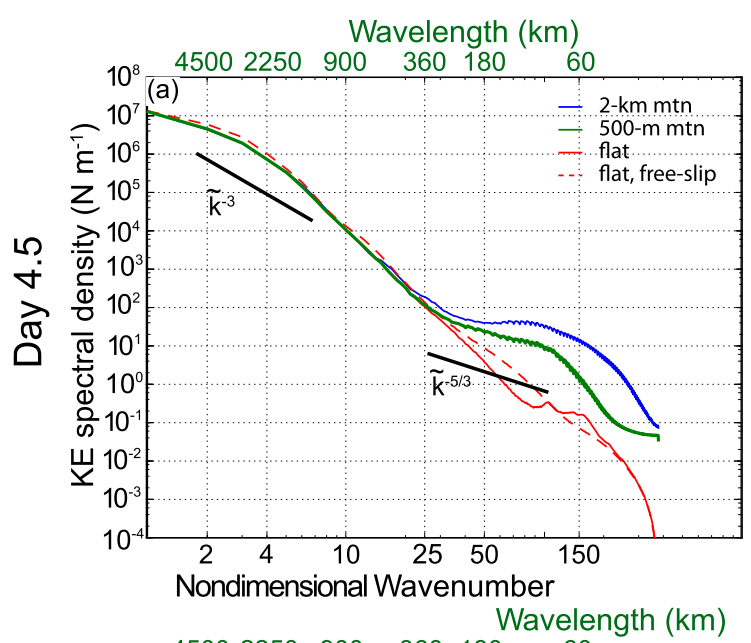

Lower Stratosphere


FIG. 5. Horizontal KE spectra $E_{h}\left(\mathrm{~N} \mathrm{~m}^{-1}\right)$ as a function of nondimensional wavenumber $\tilde{k}$ and wavelength $(\mathrm{km})$, averaged over (a),(c) the midtroposphere $(3.5-5.5 \mathrm{~km})$ and (b),(d) the lower stratosphere $(11.5-13.5 \mathrm{~km})$ at (a),(b) 4.5 and (c),(d) 6.5 days. Each panel shows the four simulations-H0FS (red dashed), H0 (red solid), H500 (green solid), and H2000 (blue solid)—along with reference lines having slopes of $\tilde{k}^{-3}$ and $\tilde{k}^{-5 / 3}$.

Although they did not examine the influence of mountains, previous investigators have demonstrated that the divergent component is largely responsible for the shallowing of the kinetic energy spectrum at mesoscale wavelengths (Waite and Snyder 2009, 2013; Peng et al. 2015a). Figure 6 compares spectra for $E_{h}, E_{r}$, and $E_{d}$ in the lower stratosphere at 6.5 days for all four simulations; the curves for $E_{h}$ (blue lines) are therefore identical to those plotted in Fig. $5 \mathrm{~d}$.

In the free-slip case, Fig. 6a, $E_{r}$ (green line, mostly underneath the blue $E_{h}$ curve) exceeds $E_{d}$ (red line) at all wavelengths, and the difference is very large except in the mesoscale between roughly 100 and $200 \mathrm{~km}$. The $E_{r}$ and $E_{d}$ spectra have similar slopes on the large scales that are steeper than $\tilde{k}^{-3}$, but these slopes are reduced on the mesoscale with the slope of $E_{d}$ becoming shallower that both the slope of $E_{r}$ and $\tilde{k}^{-5 / 3}$. These results are roughly similar to those obtained by Waite and
Snyder (2009, Fig. 7b) for $E_{r}$ and $E_{d}$ spectra in a free-slip baroclinic wave simulation except they found $E_{d}$ to be sufficiently energetic to exceed $E_{r}$ on the mesoscale. The values of $E_{d}$ in Waite and Snyder (2009) are probably higher relative to $E_{r}$ because they are from a later time in the development of the cyclone when scale contraction at the fronts and other inviscid processes have produced stronger gravity waves.

Surface stresses in the boundary layer hardly change $E_{r}$ from the values for the free-slip simulation, but a substantial mesoscale enhancement of $E_{d}$ in $\mathrm{H} 0$ relative to that in H0FS is immediately apparent in Fig. 6b. In H0, $E_{r}$ and $E_{d}$ again have similar steep slopes on the large scales, but $E_{d}$ flattens out and even obtains a positive slope as it crosses the $E_{r}$ curve at a wavelength of $180 \mathrm{~km}$. The overall difference in horizontal kinetic energy spectrum between $\mathrm{H} 0 \mathrm{FS}$ and $\mathrm{H} 0$ can be attributed to the difference in $E_{d}$ produced by the stronger gravity waves in $\mathrm{H} 0$. 

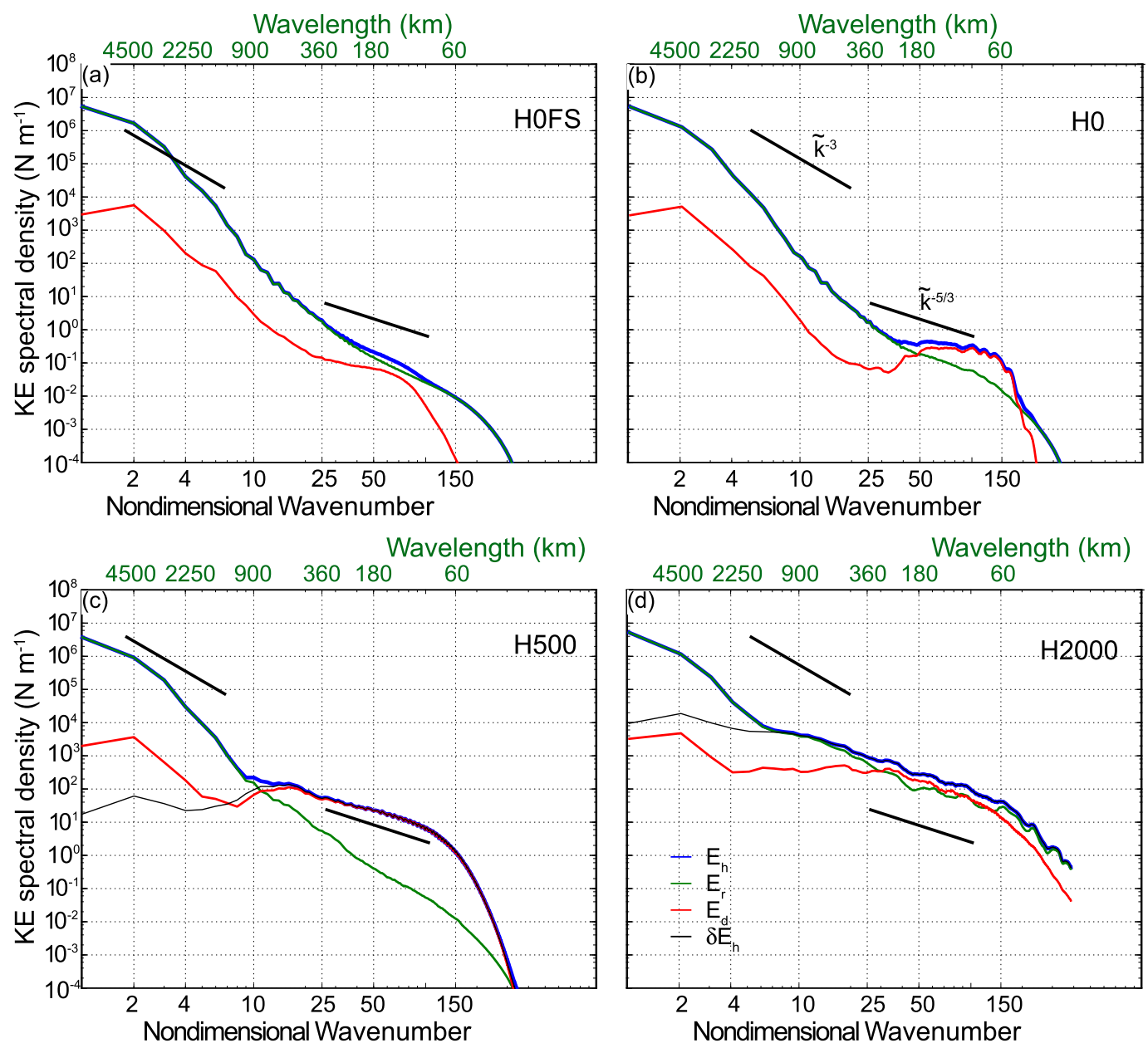

FIG. 6. Horizontal KE spectra as a function of nondimensional wavenumber $\tilde{k}$ and wavelength $(\mathrm{km})$ averaged over the lower stratosphere at 6.5 days for simulations (a) H0FS, (b) H0, (c) H500, and (d) H2000. Depicted are the total KE $E_{h}$ (blue), the rotational component $E_{r}$ (green), and the divergent component $E_{d}$ (red), along with reference lines having slopes of $\tilde{k}^{-3}$ and $\tilde{k}^{-5 / 3}$. In addition (c) and (d) show the mountain-no-mountain perturbation KE $\delta E_{h}$ (black).

The influence of an isolated 500-m-high mountain is more dramatic than the domain-wide imposition of surface stress. As shown in Fig. 6c, $E_{r}$ is only slightly more energetic in $\mathrm{H} 500$ than in $\mathrm{H} 0$, but $E_{d}$ is much stronger and its spectrum follows a $\tilde{k}^{-5 / 3}$ slope over a broad range of mesoscale wavelengths. While $E_{r}$ continues to dominate $E_{d}$ on the largest scales, $E_{d}$ becomes dominant on the mesoscale, with the cross-over point occurring at a wavelength of $900 \mathrm{~km}$. The mountain-no-mountain perturbation kinetic energy spectrum $\delta E_{h}(\tilde{k})$ is also plotted in Fig. $6 \mathrm{c}$, where $\delta E_{h}(\tilde{k})$ is computed using the same formula as for $E_{h}(\tilde{k})$ except that the horizontal velocities $\hat{\mathbf{u}}$ in (1) are replaced by the difference in the horizontal velocities between simulations with and without the mountain (in this case $\mathrm{H} 500$ and $\mathrm{H} 0$ ). At wavelengths less than $1200 \mathrm{~km}, \delta E_{h}$ is a very good approximation to $E_{d}$, suggesting that the difference in divergent kinetic energy in the two simulations is produced by vertically propagating mountain waves. Moreover, just as in $\mathrm{H} 0$, the transition in slope of the horizontal kinetic energy spectrum $E_{h}$ develops because the gravity wave generated $E_{d}$ dominates $E_{r}$ on the mesoscale.

The vertically propagating mountain waves generated by the 500 -m-high mountain do not break (MD17, Figs. 6-9a,b), but there is substantial breaking and wave-mean flow interaction when the mountain is $2 \mathrm{~km}$ high (MD17, Figs. 11, 12). ${ }^{2}$ In the 2 -km-mountain

\footnotetext{
${ }^{2}$ The figures referenced in MD17 show results on a 5-km mesh, nested inside a 15-km outer domain; those referenced in Menchaca and Durran (2018) show results on a 4-km mesh, nested inside a $12-\mathrm{km}$ outer domain. The spectra presented here were computed on a periodic $12-\mathrm{km}$ unnested domain. Nevertheless, the breaking or nonbreaking character of the waves and general structure of the wake generated by the 2-km-high mountain remains the same in all simulations.
} 
simulation, the perturbations induced by wave-breaking generate potential vorticity anomalies, some of which grow in scale as they propagate downstream (Menchaca and Durran 2018, Fig. 12), thereby providing forcing for the rotational component of the horizontal KE at intermediate or large scales. As a consequence, both $E_{d}$ and $E_{r}$ are significantly energized in $\mathrm{H} 2000$ relative to $\mathrm{H} 0$, and the mountain-no-mountain perturbation kinetic energy $\delta E_{h}$ exceeds $E_{d}$ at all wavelengths (Fig. 6d). As expected, $E_{r}$ dominates $E_{d}$ at large scales; their amplitudes become similar at wavelengths around $300 \mathrm{~km}$, but unlike the spectra in $\mathrm{H} 0$ and $\mathrm{H} 500, E_{d}$ does not dominate $E_{r}$ at shorter wavelengths. Instead $E_{r}$ and $E_{d}$ exhibit similar amplitudes down to the dissipation scale of roughly $60 \mathrm{~km}(5 \Delta x)$. The small-scale $E_{r}$ appears to be generated directly by intense small-scale vorticity perturbations within and downstream of the wave breaking regions (Menchaca and Durran 2018, Fig. 12). Similar tendencies for localized patches of turbulent flow to generate equal-amplitude divergent and rotational KE spectra were present at the anvil outflow level in simulations of isolated convective systems (Weyn and Durran 2017, Figs. 14, 15).

Several previous studies have suggested that the transition between steep spectral decay at large scales (nominally $k^{-3}$ ) and the flatter $k^{-5 / 3}$ slope on the mesoscale occurs at longer wavelengths in the stratosphere than in the troposphere because the spectral power in $E_{d}$ is nearly constant with height, while that in $E_{r}$ is distinctly lower in the stratosphere (Waite and Snyder 2009; Burgess et al. 2013; Skamarock et al. 2014). This is quite clearly the case in the H500 simulation, as illustrated by the $E_{r}$ and $E_{d}$ spectra plotted in Fig. 7 using data from day 6.5. Curves are plotted together for the midtroposphere $(3.5-5.5 \mathrm{~km})$, upper troposphere $(6.5-8.5 \mathrm{~km})$, and lower stratosphere $(11.5-13.5 \mathrm{~km})$. At wavelengths below $750 \mathrm{~km}, E_{d}$ (dashed lines) is virtually identical at all three levels. In contrast, $E_{r}$ (solid lines) is very similar in both tropospheric layers, but one or two orders of magnitude weaker in the stratosphere.

\section{c. Vertical kinetic energy spectra}

The vertical kinetic energy spectra $E_{v}$ are plotted in Fig. 8. First consider the results for the midtropospheric layer between elevations of 3.5 and $5.5 \mathrm{~km}$, shown in the left column. Similar large-scale signatures from the baroclinic wave are present in all simulations, where the slopes of $E_{v}$ evolve toward $\tilde{k}^{-3}$ at 6.5 days (Fig. 8c). This slope is also seen over the large scales in the $E_{v}$ spectra for the dry baroclinic wave simulation of Peng et al. (2015a). At smaller scales, $E_{v}$ decreases monotonically with decreasing wavelength in the H0FS simulation, whereas the corresponding spectra for $\mathrm{H} 0$ show a clear

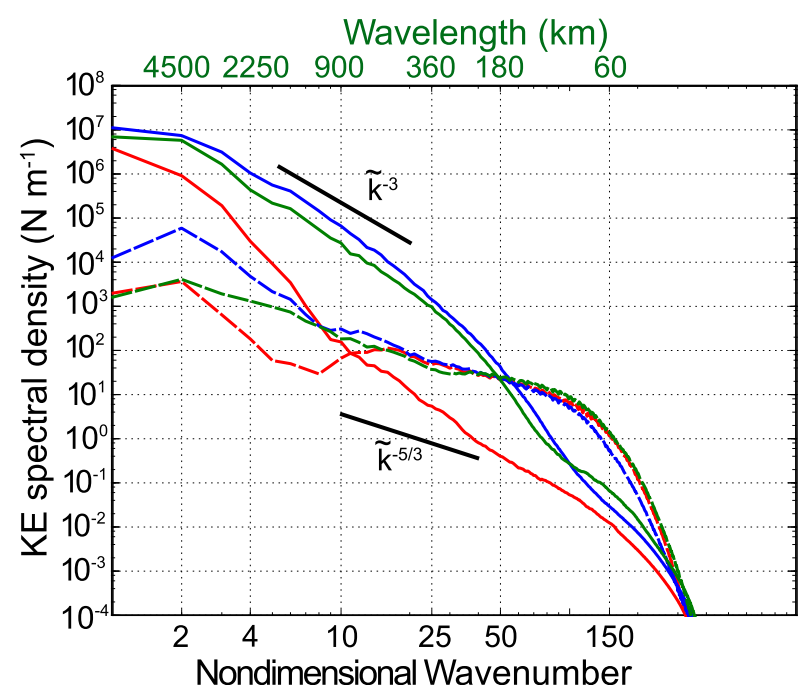

FIG. 7. Rotational and divergent components of the horizontal KE spectra as a function of nondimensional wavenumber $\tilde{k}$ and wavelength $(\mathrm{km})$ at 6.5 days for the H500 simulation. Parameters $E_{r}$ (solid) and $E_{d}$ (dashed) are averaged over the midtroposphere (3.5-5.5 km, green), the upper troposphere $(6.5-8.5 \mathrm{~km}$, blue), and the lower stratosphere $(11.5-13.5 \mathrm{~km}, \mathrm{red})$. Reference lines having slopes of $\tilde{k}^{-3}$ and $\tilde{k}^{-5 / 3}$ are also shown.

separation between the values of $E_{v}$ associated with the cyclone at large scales and a gravity-wave-produced secondary maximum in $E_{v}$ at wavelengths between $90 \mathrm{~km}$ and those shorter scales at which numerical viscosity reduces the energy. As was the case with the horizontal $\mathrm{KE}$, the waves triggered by the isolated ridge in $\mathrm{H} 500$ and $\mathrm{H} 2000$ produce much more mesoscale $E_{v}$ than does surface stress acting alone in H0. At day 6.5, the $E_{v}$ spectrum in both $\mathrm{H} 500$ and $\mathrm{H} 2000$ exhibits a modest minimum around a wavelength of $900 \mathrm{~km}$ and then gradually intensifies at shorter wavelengths. The H500 spectrum at this time is similar to that obtained (Skamarock et al. 2014, Fig. 10) in a global model with $15-\mathrm{km}$ resolution that included both terrain and moist convection. Similar, relatively flat $E_{v}$ spectra were generated by convection in simulations of a moist baroclinic wave (Peng et al. 2015a) and in 2.8-km-resolution ensemble simulations of warm-season high-precipitation events over Europe (Bierdel et al. 2012). In comparison to day 6.5 , at day 4.5 the mesoscale-wavelength values of $E_{v}$ are weaker and the local minimum between the large and small scales is much more distinct (Fig. 8a).

Because it lies above the strongest baroclinically unstable circulations, the large-scale $E_{v}$ in the lowerstratospheric layer between 11.5 and $13.5 \mathrm{~km}$ (Figs. 8b,d) is over an order of magnitude smaller than in the midtroposphere. In contrast, the gravity waves generated by the terrain or surface friction yield mesoscale $E_{v}$ whose amplitudes in the lower stratosphere are closer those in 
Mid Troposphere

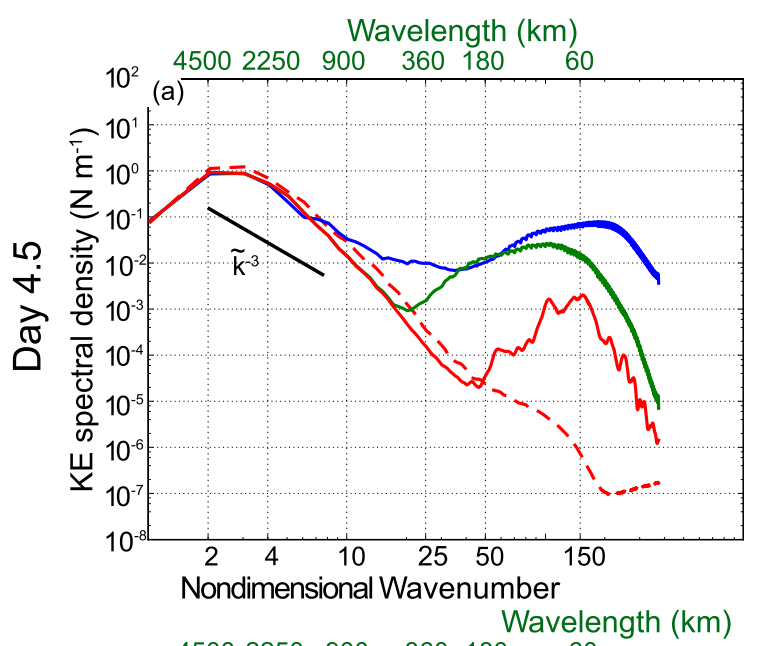

$10^{2}$ (c) $\begin{array}{lllll}4500 & 2250 \quad 900 \quad 360 & 180 & 60\end{array}$

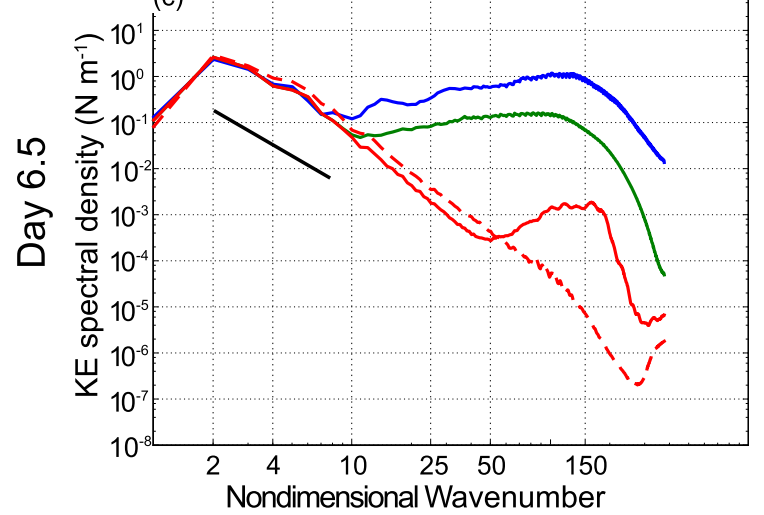

Lower Stratosphere

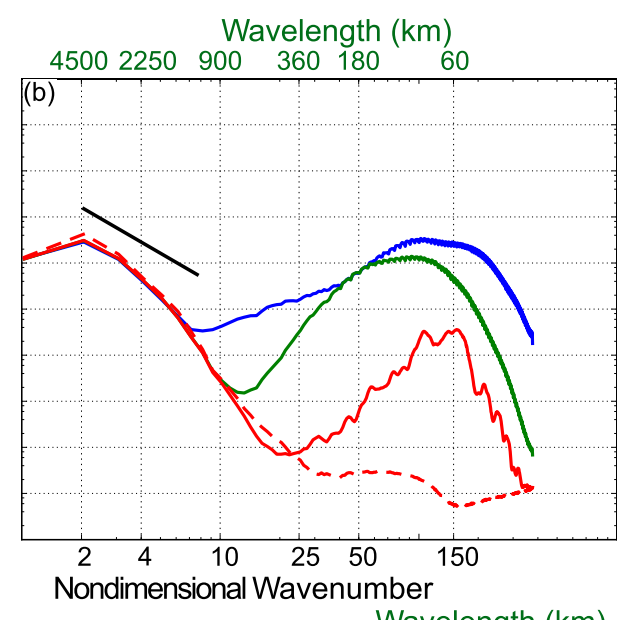

(d)

Wavelength $(\mathrm{km})$

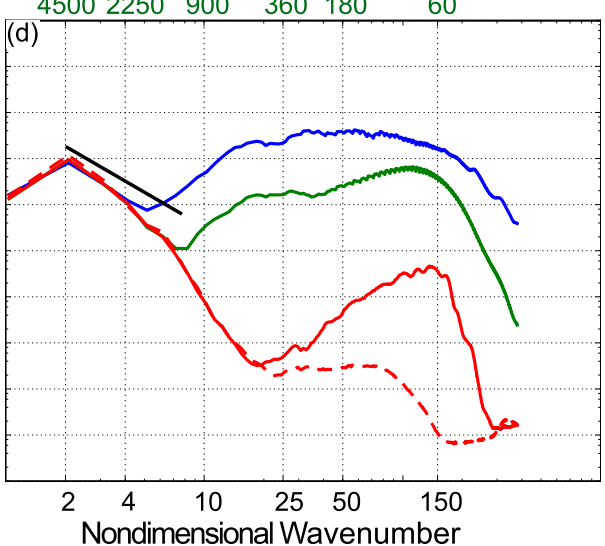

FIG. 8. As in Fig. 5, but the vertical KE spectrum $E_{v}$ with $\tilde{k}^{-3}$ reference lines.

the midtroposphere. At intermediate wavelengths, the relative minimum in $E_{v}$ between the large scale and the mesoscale becomes more pronounced in the stratosphere than in the midtroposphere. At all times and levels, the terrain and the surface stress are more pronounced sources of small-scale power for the $E_{v}$ spectra than for $E_{h}$ (cf. Figs. 5 and 8).

\section{Spectral energy budgets}

As demonstrated in the previous section, terraininduced gravity waves have a strong influence on the $\mathrm{KE}$ spectra, energizing the spectrum at mesoscale wavelengths and tending to produce a $k^{-5 / 3}$ slope on the mesoscale. In the following we analyze the spectral energy budget for $E_{h}(\mathbf{k})$ to help identify the processes governing the evolution of the horizontal KE spectra, particularly at mesoscale wavelengths. As detailed in the appendix, the spectral horizontal energy budget equation may be expressed (Peng et al. 2014, 2015a; Sun et al. 2017)

$$
\frac{\partial}{\partial t} E_{h}(\mathbf{k})=T(\mathbf{k})+B(\mathbf{k})+V(\mathbf{k})+D(\mathbf{k}),
$$

where $T$ is the contribution from advection written in the form (A8) that is conservative in the sense that it integrates to zero over all $\mathbf{k}, B$ is the buoyancy forcing, which represents the conversion from available potential energy (APE) to horizontal kinetic energy, $V$ is the divergence of the vertical KE flux, and $D$ is dissipation.

We are particularly interested in examining the extent to which $\mathrm{KE}$ is cascading between scales, and for such purposes it is convenient to plot the contributions from the forcing terms in (6) as the cumulative sum over all 2D wavenumbers larger than a given $k$ (Augier and Lindborg 2013; Malardel and Wedi 2016). For an arbitrary variable $X(k)$ we can define

$$
\Pi_{X}(k)=\sum_{k \leq l \leq N} X(l),
$$

where $N$ is the maximum 2D wavenumber represented on the grid. If $X(k)$ is one of the source terms in (6) and if 

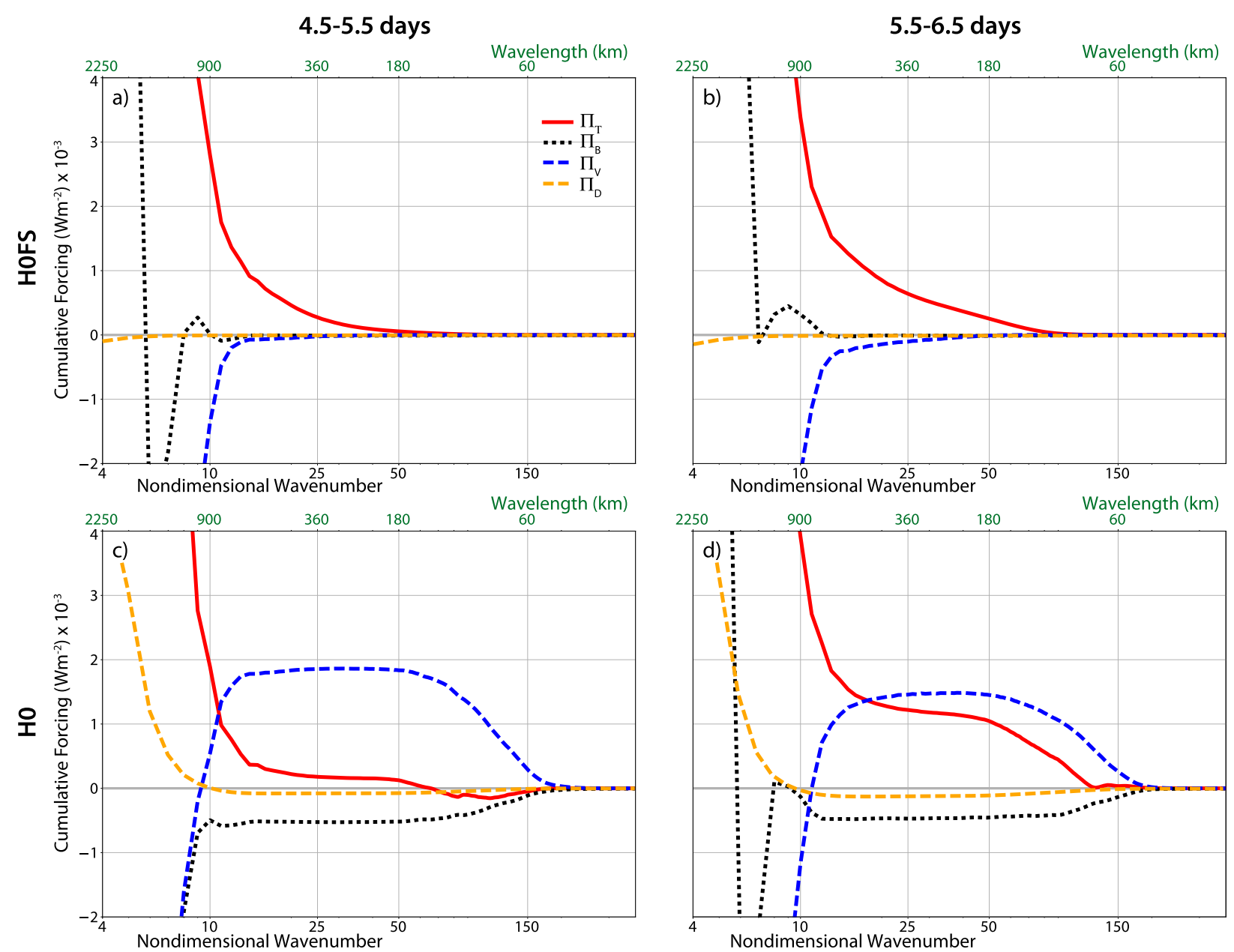

FIG. 9. Cumulative spectral budget terms averaged over the lower stratosphere (11.5-13.5 km) for (a),(b) H0FS and (c),(d) H0 averaged over (a),(c) 4.5-5.5 and (b),(d) 5.5-6.5 days. Plotted are $\Pi_{T}$ (red solid), $\Pi_{B}$ (black dotted), $\Pi_{V}$ (blue dashed), and $\Pi_{D}$ (orange dashed).

$\Pi_{X}(k)$ is plotted as a function of $k$, then the points in this plot where $\Pi_{X}$ has a negative slope $\left(\partial \Pi_{X} / \partial k<0\right)$ correspond to wavenumbers at which $X(k)$ is tending to increase $E_{h}(k)$ (Malardel and Wedi 2016, Fig. 3). Similarly, points with a positive slope correspond to wavenumbers at which $X(k)$ acts to decrease $E_{h}(k)$. Because $T(k)$ describes conservative energy transfer between horizontal wavenumbers, the sign of $\Pi_{T}$ indicates whether it is acting to transfer $E_{h}$ upscale or downscale. As an example, if $\Pi_{T}(k)>0, T(k)$ is acting to produce a net transfer of $E_{h}$ to wavenumbers greater than $k$; which would be a downscale transfer to shorter wavelengths. Similarly, negative values of $\Pi_{T}$ represent upscale transfer. As before, the values of $\Pi_{X}$ will be plotted as a function of the nondimensional discrete horizontal wavenumber $\tilde{k} \equiv\left(L_{y} / 2 \pi\right) k$.

\section{a. Lower stratosphere}

The terms in the cumulative spectral KE budget, averaged over the lower stratospheric layer $11.5 \leq z \leq 13.5 \mathrm{~km}$, are plotted for the simulations without topography in Fig. 9. The longest wavelengths are omitted in these plots for better visibility, because 1) our focus in not on baroclinic instability, but rather on mesoscale processes, 2) the magnitudes of the terms are typically off-scale at the longest wavelengths, and 3) because the resolution in wavenumber space is very coarse at the longest wavelengths. ${ }^{3}$

In the free-slip case HOFS, downscale advective transfers are the primary source of KE at scales less than about $750 \mathrm{~km}\left(\Pi_{T}>0, \partial \Pi_{T} / \partial \tilde{k}<0\right.$, Figs. 9a,b). In the 5.5-6.5-day average, there is also a tendency for the vertical energy flux divergence to remove KE from the layer $\left(\partial \Pi_{V} / \partial \tilde{k}>0\right)$. The contribution from dissipation $\Pi_{D}$ is negligible.

\footnotetext{
${ }^{3}$ Nevertheless, as expected, $\Pi_{T}(0)=0$.
} 
The budget for $\mathrm{H} 0$ is quite different: vertical energy flux convergence acts to increase $\mathrm{KE}$ over wavelengths of $50 \leq \lambda \leq 180 \mathrm{~km}\left(\partial \Pi_{V} / \partial \tilde{k}<0\right.$, Figs. $\left.9 \mathrm{c}, \mathrm{d}\right)$. This forcing is opposed by buoyancy forces converting KE to PE over wavelengths of $50 \leq \lambda \leq 150 \mathrm{~km}$, which is roughly the range over which there are significant differences in the lower stratospheric KE spectra of $\mathrm{HOFS}$ and $\mathrm{HO}$ at 4.5 days in Fig. 5b. The 4.5-5.5-day forcing indicated by $\Pi_{T}$ is weak, and the advective energy transfer switches from downscale to upscale for wavelengths shorter than about $130 \mathrm{~km}$. Even at wavelengths longer than $130 \mathrm{~km}$, the presence of boundary layer dissipation does not increase the contribution from $T(k)$ relative to the freeslip case, although there is a region of $200<\lambda<400 \mathrm{~km}$ in Fig. $9 \mathrm{c}\left(\partial \Pi_{T} / \partial \tilde{k} \approx 0\right)$ through which $\mathrm{KE}$ in transferred downscale without deposition. In contrast, in the 5.56.5-day averaged $\mathrm{H} 0$ budget there is pronounced downscale advective transfer of $\mathrm{KE}$ at all wavelengths and a strong tendency for $T(k)$ to increase KE in the range $70 \leq \lambda \leq 180 \mathrm{~km}$. The wavelength range in Fig. $5 \mathrm{~d}$ where $\mathrm{H} 0$ deviates from $\mathrm{HOFS}$ is roughly the same range where there is significant contributions from $\Pi_{V}, \Pi_{T}$, or $\Pi_{B}$ in the 5.5-6.5-day cumulative budget. The contribution from $\Pi_{D}$ is very small compared to the other terms, but surprisingly, it is greatest at large scales, and it acts to increase the KE at those scales. The tendency to increase KE comes entirely from the vertical component of the eddy diffusivity [see (A14)], and represents the convergence of vertical diffusive fluxes. ${ }^{4}$

The horizontal KE in the H500 case is strongly forced by vertical energy flux convergence (Figs. 10a,b), which is to be expected based on linear theory for steady-state mountain waves. ${ }^{5}$ As derived by Eliassen and Palm (1960), the momentum flux carried by steady linear 2D mountain waves in the $x-z$ plane is constant with height,

$$
\frac{d}{d z}\left(\bar{\rho} \int u^{\prime} w^{\prime} d x\right)=0
$$

and the ratio of the vertical energy flux to the momentum flux is determined by the basic state cross-mountain wind speed $U(z)$ such that

$$
\int p^{\prime} w^{\prime} d x=-U \bar{\rho} \int u^{\prime} w^{\prime} d x
$$

\footnotetext{
${ }^{4}$ It is not clear why the dissipation so strongly forces the longest wavelengths, but it is worth repeating that, at these long wavelengths, the magnitude of $\Pi_{D}$ is still far smaller than that of the other three terms.

${ }^{5}$ Wave action, not perturbation KE, is conserved as gravity waves propagate through a shear flow.
}

where in the preceding, the primes denote perturbations, $p$ is pressure, and the integrals are either taken over a wavelength or the full periodic domain. The relation (9) also holds in observed mountain waves (Smith et al. 2008, 2016). Since the momentum flux associated with the mountain waves in the H500 simulation is almost constant with height (Durran and Menchaca 2019, Fig. 4), (9) implies that the energy flux associated with such waves would decrease with height at levels above the jet, where the environmental wind speed decreases with height. Such vertical energy flux convergence, which acts to increase $\mathrm{KE}$, is strongest in the wavelength range $60<\lambda<180 \mathrm{~km}$, particularly at 5.5-6.5 days when $\partial \Pi_{V} / \partial \tilde{k}$ is strongly negative.

Over both the 4.5-5.5- and 5.5-6.5-day averaging periods, $T(k)$ is transferring energy upscale across wavelengths in the range $90<\lambda<1500 \mathrm{~km}$. This is roughly similar to the $90<\lambda<1100-\mathrm{km}$ range of wavelengths over which the KE spectrum for H500 exhibits a clean $\tilde{k}^{-5 / 3}$ slope in Fig. $5 \mathrm{~d}$. KE is removed by advective transfer from almost the same small scales where it is forced by vertical energy flux divergence, $70<\lambda<150 \mathrm{~km}$. Conversely, $T(k)$ deposits KE at wavelengths greater than about $1100 \mathrm{~km}$, where $V(k)$ is acting to reduce KE. Downscale advective transfer is also a source of $\mathrm{KE}$ at small wavelengths less than $70 \mathrm{~km}$. Vertically propagating gravity waves have zero buoyancy flux, so not surprising that $\Pi_{B}$ is not an important term in the budget. The dissipation $\Pi_{D}$ is also unimportant.

The overall scenario suggested by the lowerstratospheric KE budget from the H500 simulation is that vertical energy flux divergence associated with gravity waves propagating from below deposits energy at small scales, which then cascades to larger scales via conservative advection. Since, at wavelengths shorter than about $800 \mathrm{~km}$, the $\mathrm{KE}$ is entirely divergent and equal to difference $\delta \mathrm{KE}_{h}$ between the $\mathrm{KE}$ in the mountain and no-mountain simulations (Fig. 6c), the inertial cascade is likely associated with gravity waves. The upscale cascade is surprising because gravity waves are thought to cascade energy downscale to create the $k^{-5 / 3} \mathrm{KE}$ spectrum in the ocean (Garrett and Munk 1979). Other recently computed lower stratospheric spectral KE budgets suggest a variety of alternative behaviors when there is forcing from parameterized moist convection. Augier and Lindborg (2013) found a downscale advective cascade across the mesoscale in AFES simulations, but very little advective transfer in the ECMWF IFS model. On the other hand, Peng et al. (2015a) found a weak upscale energy transfer by $\Pi_{T}$ at wavelengths longer than $360 \mathrm{~km}$ in a channel-model simulation of moist baroclinic instability. Even increasing the mountain height to $2 \mathrm{~km}$ in these simulations 

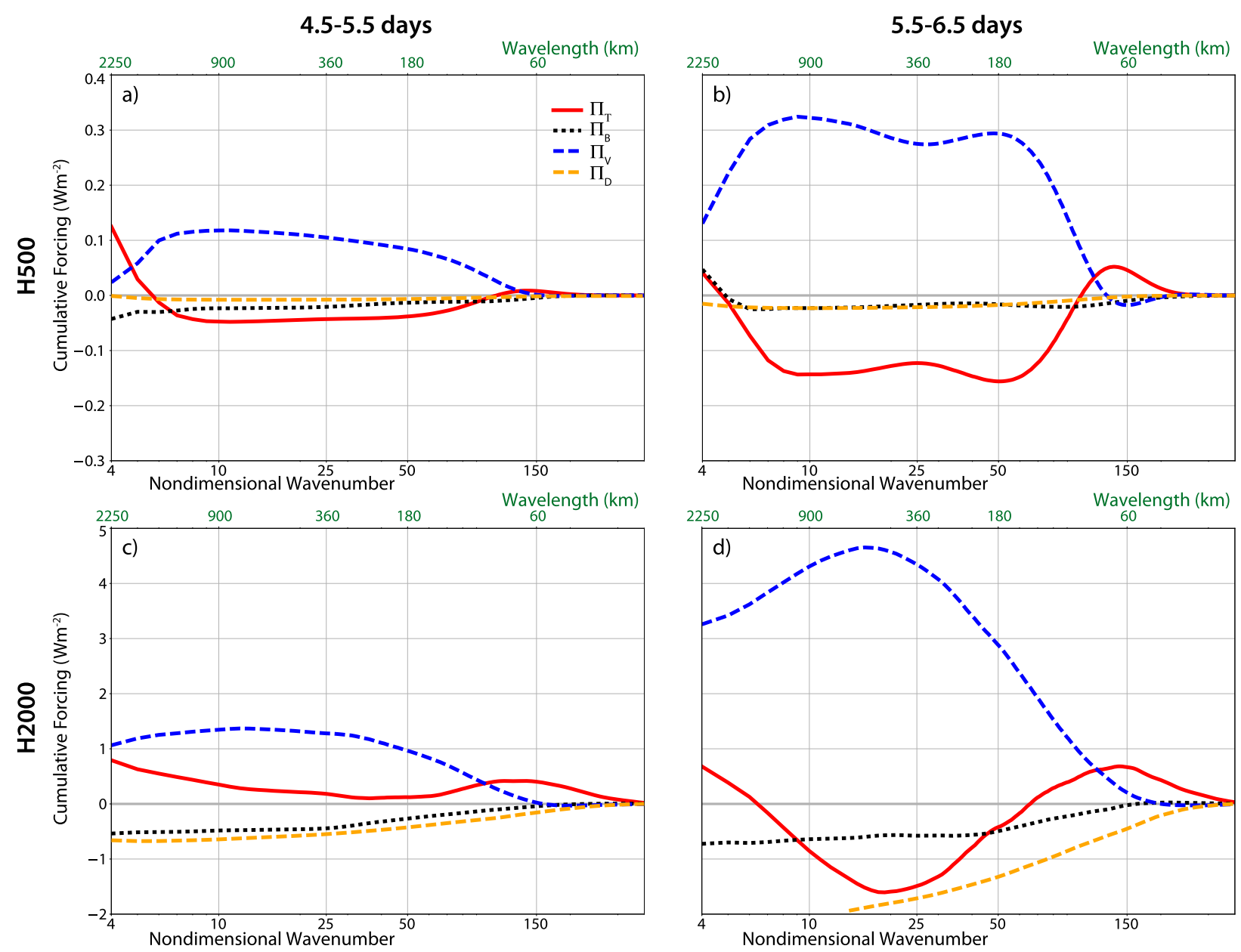

FIG. 10. As in Fig. 9, but for (a),(b) H500 and (c),(d) H2000. Note the increases in the scale on the vertical axis as the mountain height is increased.

changes the direction of the KE transfer associated with $\Pi_{T}$ at $4.5-5.5$ days to weakly downscale.

In contrast to the H500 simulation, extensive wave breaking, turbulent mixing, and associated secondary circulations occur in the presence of the 2-km-high mountain, although there is no wave breaking in the stratosphere until after day 4.5 (MD17). As a consequence, the cumulative buoyancy forcing in $\mathrm{H} 2000$ increases by more than an order of magnitude from that found in H500 (Figs. 10c,d, note the increase the vertical scale from Figs. 10a,b): $B(k)$ tends to convert KE to available potential energy at essentially all wavelengths longer than $60 \mathrm{~km}$, but the effect is strongest up through wavelengths of $360 \mathrm{~km}$ in the 4.5-5.5-day average and up through wavelengths of $180 \mathrm{~km}$ in the 5.5-6.5-day average. At 4.5-5.5 days, the distribution of $\Pi_{V}$ is roughly similar to that for $\mathrm{H} 500$, but at 5.5-6.5 days, there is a strong tendency for the vertical energy flux divergence to increase the KE over the larger range of wavelengths $(50<\lambda<500 \mathrm{~km})$, while acting to decrease the $\mathrm{KE}$ at longer wavelengths. Large differences from the H500 case are also apparent in $\Pi_{T}$. At 4.5-5.5 days, upscale energy transfer has been replaced by downscale transfer and $T(k)$ also tends to increase the $\mathrm{KE}$ at all wavelengths longer than $300 \mathrm{~km}$.

Finally, in contrast to the other cases, $\Pi_{D}$ is nontrivial at 4.5-5.5 days in $\mathrm{H} 2000$, where it acts as a sink of KE at a rate similar to $\Pi_{B}$ over all wavelengths. At 5.56.5 days, $\Pi_{D}$ acts as an even stronger sink on the KE (Fig. 10d). One might suppose that the dissipative forcing would be focused at the highest wavenumbers, and the contribution to $\partial \Pi_{D} / \partial k$ from horizontal eddy diffusion is indeed limited to wavelengths shorter than $180 \mathrm{~km}$. The contribution from vertical eddy diffusion, on the other hand, acts across a much larger range of scales. One reason why forcing from $\Pi_{D}$ appears across a broad range of scales is because the regions of vigorous subgrid-scale mixing produced by wave breaking are 
4.5-5.5 days
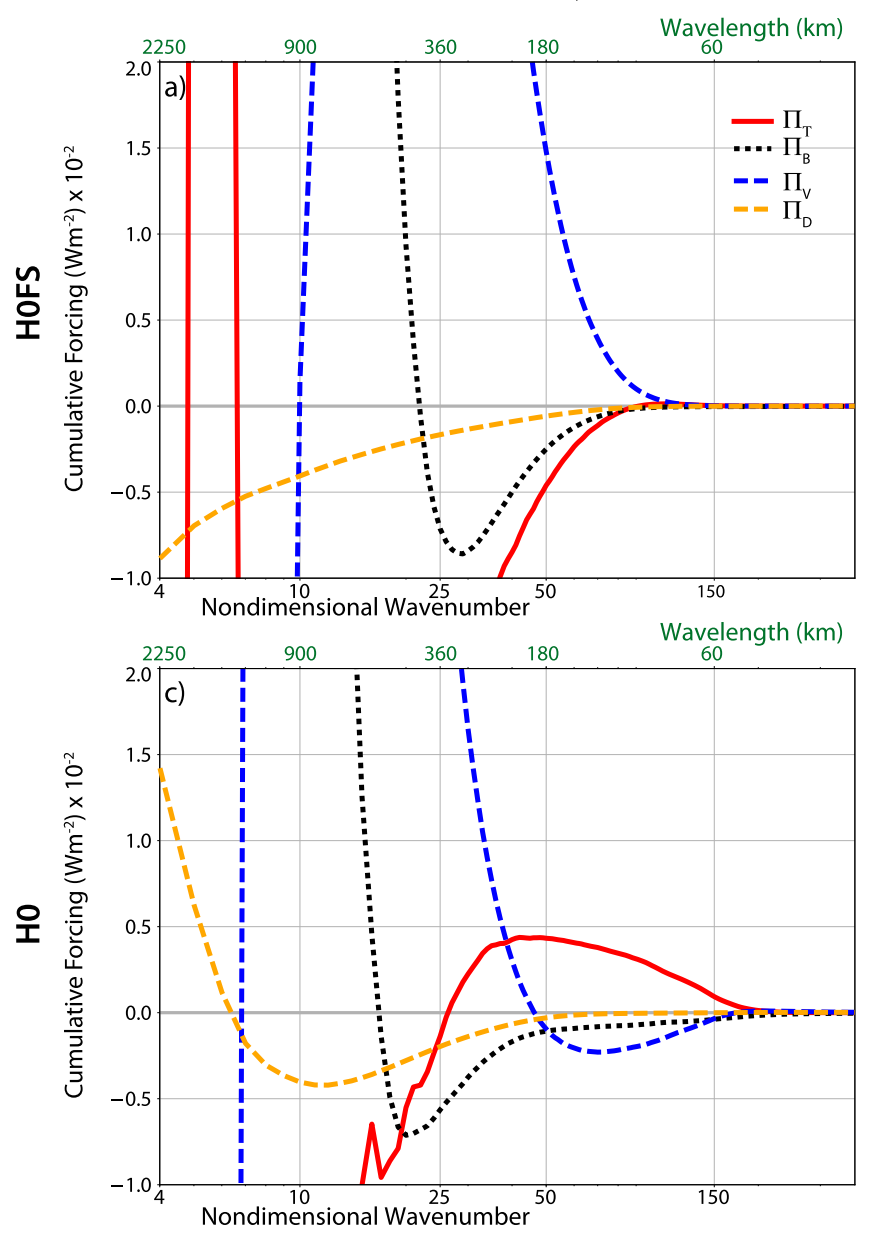

5.5-6.5 days
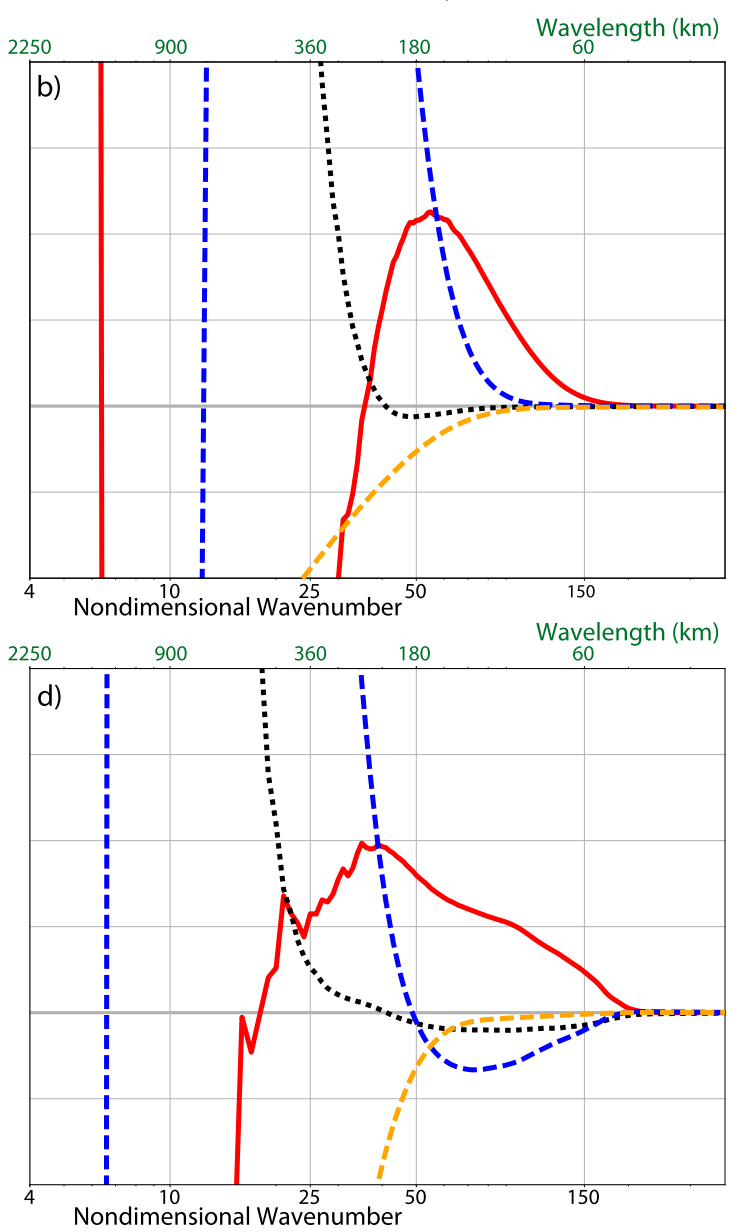

FIG. 11. As in Fig. 9, but averaged over the midtroposphere $(3.5-5.5 \mathrm{~km})$.

localized in physical space to a small region above the mountain, and as such, the distribution of their dissipative forcing is very broad in wavenumber space. Peng et al. (2015a) found a roughly similar wavenumber dependence for the dissipation in the lower stratosphere in their moist (RH60) baroclinic wave simulation.

As with the other terms in the cumulative budget, the differences in $\Pi_{T}$ between $\mathrm{H} 500$ and $\mathrm{H} 2000$ are greatest in the 5.5-6.5-day average (cf. Figs. 10b and 10d). Instead of a broad region where $\partial \Pi_{T} / \partial \tilde{k}$ is near zero, the conservative advective flux largely counteracts the forcing from $V(k)$, tending to decrease $\mathrm{KE}$ in the wavelength range $50<\lambda<500 \mathrm{~km}$ while acting to increase $\mathrm{KE}$ at longer and shorter wavelengths. The cumulative advective flux is upscale for $150<\lambda<1500 \mathrm{~km}$ and downscale at longer and shorter wavelengths. Given the broad range of wavelengths over which direct forcing is implied by significant variations in $\Pi_{V}, \Pi_{T}$, and $\Pi_{D}$, it is apparent that a nearly nondissipative advective cascade of $\mathrm{KE}$, such as that found for $\mathrm{H} 500$, is not required to produce the $\tilde{k}^{-5 / 3}$ spectral slope shown for H2000 in Fig. 5d over wavelengths in the range $60<\lambda<1500 \mathrm{~km}$.

\section{b. Midtroposphere}

We now consider the cumulative spectral horizontal $\mathrm{KE}$ budget for the $3.5-5.5-\mathrm{km}$ layer in the middle troposphere. In both HOFS and H0, a negative $\Pi_{B}$ slope at synoptic scales (Fig. $11, \Pi_{B}$ itself is largely off scale) is associated with the conversion of APE to KE as expected in a baroclinically unstable flow (Augier and Lindborg 2013; Malardel and Wedi 2016; Peng et al. 2015b). In the mesoscale, the 4.5-5.5-day-averaged budget for H0FS (Fig. 11a) shows horizontal KE being created by vertical energy flux divergence $V(k)$, while it is removed by both $B(k)$ and $T(k)$ between roughly $90<\lambda<360 \mathrm{~km}$, and transferred upscale. In comparison, at 5.5-6.5 days the tendency of $B(k)$ to diminish $\mathrm{KE}$ is much smaller, while $T(k)$ acts more strongly to reduce $\mathrm{KE}$ at scales greater than $150 \mathrm{~km}$, and to increase $\mathrm{KE}$ at shorter scales (Fig. 11b). The advective energy transfer 

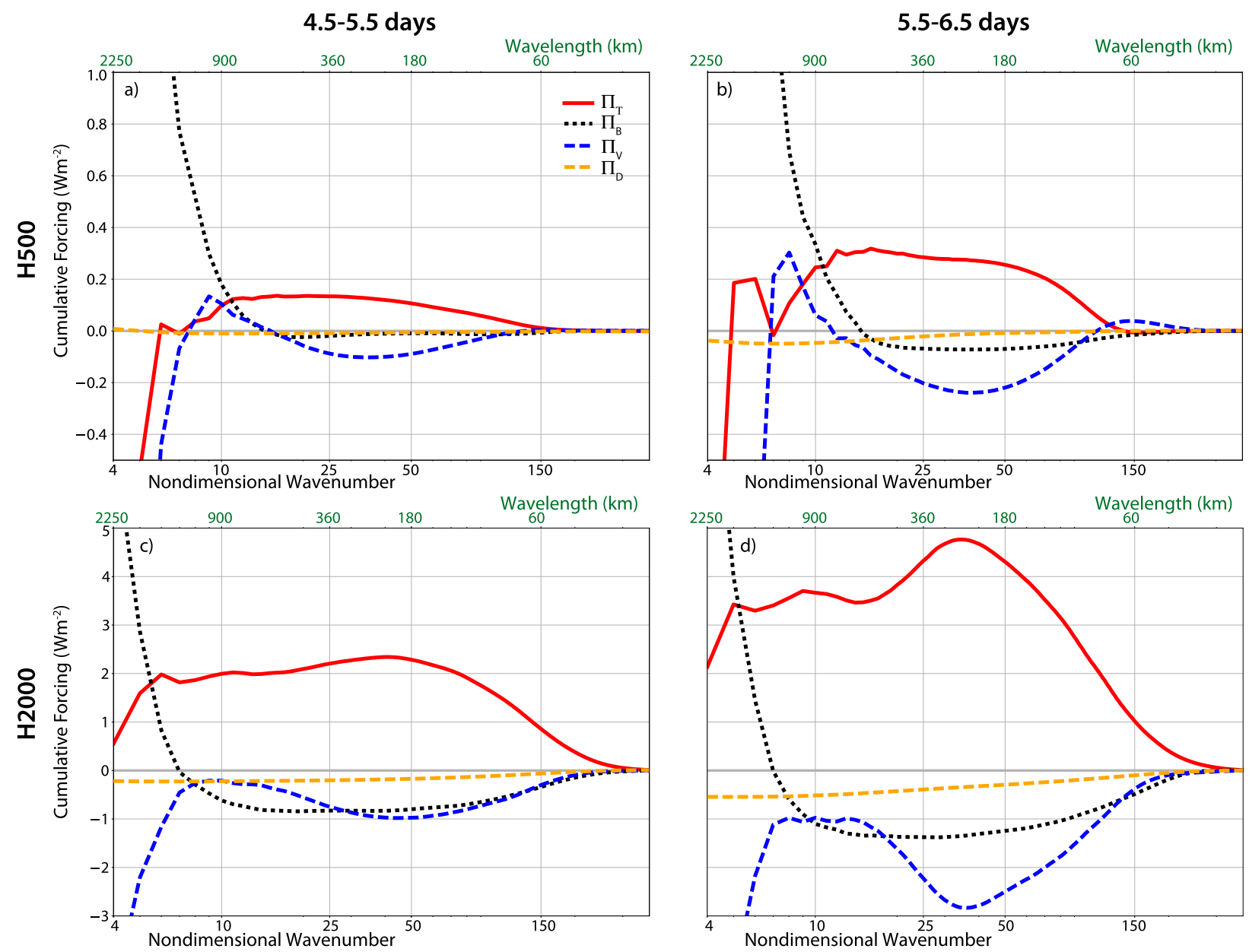

FIG. 12. As in Fig. 10, but averaged over the midtroposphere $(3.5-5.5 \mathrm{~km})$. Note the further increases in the scale on the vertical axis.

is downscale for $\lambda<250 \mathrm{~km}$ and upscale for longer mesoscale wavelengths. The vertical scale is sufficiently exaggerated (to show the mesoscale region) that $\Pi_{D}$ looks potentially nontrivial, but in fact it is not significant in comparison to the dominant budget terms.

Under the presence of surface stress, $V(k)$ acts to remove KE over wavelengths between $50<\lambda<150 \mathrm{~km}$, which are approximately the same wavelengths at which vertical energy flux divergence in the same $\mathrm{H} 0$ simulation supplies KE to the lower stratosphere (cf. Figs. 9c,d and $11 \mathrm{c}, \mathrm{d})$. This negative KE tendency is largely balanced by downscale advective transport of KE beginning around wavelengths of $220 \mathrm{~km}$. The region with a spectral slope approximating $\tilde{k}^{-5 / 3}$ in the midtroposphere at day 6.5 in Fig. $5 \mathrm{c}$ also occupies most of this same wavenumber band. The contribution of $\Pi_{D}$ to the spectral KE budget for $\mathrm{H} 0$ is again small compared to the dominant terms in the budget, although as was the case in the lower stratosphere, vertical subgrid scale diffusive fluxes make a positive contribution to the $\mathrm{KE}$ at very long wavelengths.
Turning to the influence of topography, in the midtroposphere the contributions of $V(k)$ and $T(k)$ in H500 are roughly opposite those in the lower stratosphere at wavelengths shorter than $250 \mathrm{~km}$. Since the budget is computed below the height of the jet maximum, (9) suggests steady nonbreaking mountain waves will produce energy flux divergence. As shown in Figs. 5a and 5c, over approximately the same $90<\lambda<180 \mathrm{~km}$ interval, where H500 exhibits a $\tilde{k}^{-5 / 3}$ spectrum the vertical energy flux divergence acts to reduce $\mathrm{KE}$, and the conservative advective transfer is downscale and tending to increase the KE (Figs. 12a,b). Nevertheless, at scales larger than the mountain waves $(250<\lambda<1100 \mathrm{~km}) V(k)$ exhibits a weak tendency to increase KE, which is similar to the behavior in the lower stratosphere. The buoyancy forcing $B(k)$ plays about the same modest role in both the midtroposphere and the lower stratosphere for wavelengths less than $450 \mathrm{~km}$ (cf. Figs. 10a,b and 12a,b), but at longer wavelengths there is substantial conversion of APE to KE in 
the growing baroclinically unstable wave. The contributions from dissipation are negligible.

In $\mathrm{H} 2000$ the midtropospheric $\Pi_{V}$ shows a tendency for $V(k)$ to reduce $\mathrm{KE}$ at mesoscale wavelengths shorter than $230 \mathrm{~km}$, which is again opposite its behavior in the lower stratosphere (Figs. 12c,d). The advective KE transfer is downscale at all wavelengths and tends to increase $\mathrm{KE}$ for $\lambda<230 \mathrm{~km}$. At slightly larger scales, the slopes of $\Pi_{V}$ and $\Pi_{T}$ switch signs; particularly in the 5.5-6.5-day average, there is a tendency for $V(k)$ to increase, and $T(k)$ to decrease, $\mathrm{KE}$ in the wavelength band of $230<\lambda<750 \mathrm{~km}$. At wavelengths less than $180 \mathrm{~km}$, $B(k)$ tends to more strongly decrease KE than in any of the other cases. As in all the midtropospheric budgets, at synoptic-scale wavelengths $B(k)$ tends to increase KE in the baroclinically unstable flow. The net effect of all this forcing at mesoscale wavelengths is to increase the 6.5-day horizontal $\mathrm{KE}$ relative to that in the $\mathrm{H} 0 \mathrm{simu}$ lation at all wavelengths shorter than $900 \mathrm{~km}$ (Fig. 5c), but instead of a $\tilde{k}^{-5 / 3}$ slope over these wavelengths, the spectrum follows an approximate $\tilde{k}^{-3}$ slope. Because there is almost no wavebreaking in the midtroposphere, the dissipative contribution of dissipation $\Pi_{D}$ to the spectral KE budget is much weaker, than it is in the lower stratosphere, and this difference is particularly pronounced at 5.5-6.5 days (cf. Figs. 10d and 12d).

Also of note is the systematic increase in the scale at which the conversion of APE to KE ceases as the topographic forcing increases. In the 5.5-6.5-day average, $\Pi_{B}$ exhibits strong negative slopes down through wavelengths of about $360 \mathrm{~km}$ in $\mathrm{H} 0,750 \mathrm{~km}$ in H500, and $900 \mathrm{~km}$ in H2000 (Figs. 11d, 12b,d). The mountains appear to be inhibiting the cyclogenetic intensification of $\mathrm{KE}$ at small synoptic scales.

\section{Conclusions}

We have examined the influence of gravity waves on the KE spectrum associated with a developing cyclone in a dry baroclinically unstable shear flow. Although deviations from geostrophic balance eventually become strong enough to trigger gravity wave emission in freeslip simulations of such cyclones (Waite and Snyder 2009), we have focused on somewhat earlier stages of the cyclone life cycle when the gravity waves triggered in the free-slip simulation are weak. In comparison to the free-slip case, the gravity waves in the vicinity of the cold front are significantly enhanced by surface stress. The precise generation mechanism for these waves, and the extent to which they are accurately captured in the numerical simulations, are left for future analysis. The surface-stress-induced gravity waves energize the divergent component $E_{d}$ of the horizontal KE spectrum at wavelengths less than about $200 \mathrm{~km}$, but they have almost no influence on the rotational component $E_{r}$. The total horizontal KE spectrum in both the troposphere and the stratosphere develops a pronounced region of shallow slope as a result of these frictionally generated gravity waves. The simulations with topography also incorporated the same surface boundary layer parameterization to avoid unrealistically large surface winds and high-amplitude mountain waves. Although the terrain only covers about $0.1 \%$ of the surface area filled by significant large-scale disturbances at 6.5 days, mountain waves generated by flow over the single isolated ridge have a major impact on the domain-integrated KE spectrum, and this impact is much greater than that generated by surface stress alone.

The mountain waves generated by the 500-m-high ridge do not break and their contribution to the horizontal $\mathrm{KE}$ spectrum $E_{h}$ is entirely captured by the divergent component $E_{d}$. When the waves are mature, at 6.5 days, the slope of the $E_{d}$ spectrum closely matches $k^{-5 / 3}$, and the amplitude of $E_{d}$ is constant with height between the midtroposphere and lower stratosphere at wavelengths shorter than about $750 \mathrm{~km}$. The transition from a $k^{-3}$ slope at large scales to a $k^{-5 / 3}$ slope at small scales occurs at longer wavelengths in the stratosphere than in the troposphere because of the lower level of background $E_{r}$ in the stratosphere, as previously suggested by Waite and Snyder (2009), Burgess et al. (2013), and Skamarock et al. (2014).

The mountain waves generated by the $2-\mathrm{km}$-high ridge break down in both the troposphere and the stratosphere (MD17), creating patches of vertical vorticity, and thereby enhancing the rotational as well as the divergent components of $E_{h}$ at essentially all scales. In the stratosphere at day $6.5, E_{r}$ and $E_{d}$ have roughly equal amplitude over wavelengths $60<\lambda<360 \mathrm{~km}$, and their sum $E_{h}$ has developed an approximate $k^{-5 / 3}$ slope over the very broad spectral band of $60<\lambda<1800 \mathrm{~km}$. In the troposphere, on the other hand, where $E_{r}$ is largely energized by baroclinic instability, the flattening of the $E_{h}$ spectral slope is much less pronounced. The creation of patches of vertical vorticity in wave breaking regions is at least qualitatively comparable to circulations generated in simulations of deep convective systems in horizontally homogeneous environments [compare Fig. 13a of Menchaca and Durran (2018) with Figs. 17d-f of Weyn and Durran (2017)], and the rotational and divergent components of the horizontal KE spectrum were also found to have roughly equal amplitude in the upper troposphere and lower stratosphere in those convective systems.

Spectral budgets for the horizontal KE were evaluated in the midtroposphere and the lower stratosphere. 
The most surprising results were obtained for the 500-m-mountain case in the lower stratosphere, where the 5.5-6.5-day averaged budget shows energy input in the mesoscale from vertical energy flux convergence, energy removal at the same scale by conservative advection, and a near inertial advective cascade upscale from wavelengths of about $180 \mathrm{~km}$ to wavelengths of about $1100 \mathrm{~km}$ (Fig. 10b). Nevertheless, as apparent in the corresponding KE budget for the simulation with the 2-km-high mountain, the horizontal KE spectrum can also develop an approximate $k^{-5 / 3}$ slope over an even broader range of wavelengths without any inertial cascade whatsoever. Instead, the $k^{-5 / 3}$ slope is produced by nontrivial contributions from all four terms in the spectral KE budget: vertical energy flux convergence, conservative advection, buoyancy forcing, and dissipation (Fig. 10d). Moreover, the forcing from the two largest terms, vertical energy flux convergence and conservative horizontal advection, flips sign around a wavelength of about $500 \mathrm{~km}$ without producing any obvious deviations from the $k^{-5 / 3}$ slope. Given the previously discussed similarities between the local perturbations produced in wave breaking regions and those generated aloft by deep convection, our finding that direct forcing across a wide range of scales is responsible for the $k^{-5 / 3}$ spectrum in the 2-km-mountain simulation might have been anticipated from Sun et al. (2017), who obtained a similar result analyzing simulations of deep convection.

The contributions from vertical energy flux divergence and conservative advection in the midtroposphere $\mathrm{KE}$ budgets are opposite in sign relative to those in the lower stratospheric budgets. Vertical energy fluxes are removing $\mathrm{KE}$ from the midtroposphere at mesoscale wavelengths, transporting it upward, and depositing it in the lower stratosphere. The conservative advective transport of KE also switches from downscale in the midtroposphere to upscale in the lower stratosphere, although in the 5.5-6.5-day averaged budgets, the only near inertial cascade occurs in the lower stratosphere for the 500-m-high mountain.

The slope of the mesoscale KE spectrum varies significantly over time and does not seem to be produced by any single universal dynamical mechanism. At 6.5 days, for example, the spectral slopes at mesoscale wavelengths in the lower stratosphere are close to $k^{-5 / 3}$, but are far more variable at 4.5 days (Figs. 5 b,d). Substantial variability in the slope of the mesoscale energy spectrum has also been documented in previous studies. Selz et al. (2019) found a strong correlation between precipitation rate and variability in the spectral slope at short wavelengths in three years of analysis data over Europe. Case-study ensemble simulations of weakly forced diurnal convection have demonstrated that the slope can approach $k^{-5 / 3}$ during hours of active convection and then drop back to steeper values when the convection dies down (Weyn and Durran 2019, Fig. 5). It may be that the $k^{-5 / 3}$ mesoscale KE spectrum in the atmosphere is not as universal as sometimes supposed, and that it slope should not be interpreted as providing evidence for the action of any particular dynamical mechanism (Stumpf and Porter 2012).

Among the different ways that mountain waves produce a $k^{-5 / 3}$ spectrum, the H500 case is worthy of further comment. The mesoscale perturbations in this case are essentially all gravity waves, and the spectral energy budget suggests an inertial cascade is occurring between the large and small scales, yet in contrast to the commonly understood action of gravity waves in creating the $k^{-5 / 3} \mathrm{KE}$ spectrum in the ocean (Garrett and Munk 1979), the energy transfer is upscale (Figs. 10a,b). We hypothesize this upscale transfer is produced as the waves propagate through a background horizontal flow with strong horizontal variations. The WKB ray tracing equations for the horizontal wavenumbers $\left(k_{x}, k_{y}\right)$ in quasi-linear gravity waves propagating upward and westward relative to a background velocity $[U(x, y), V(x, y)]$ are

$$
\begin{aligned}
& \frac{D_{g} k_{x}}{D t}=-k_{x} \frac{\partial U}{\partial x}-k_{y} \frac{\partial V}{\partial x}, \\
& \frac{D_{g} k_{y}}{D t}=-k_{x} \frac{\partial U}{\partial y}-k_{y} \frac{\partial V}{\partial y} .
\end{aligned}
$$

Chen et al. (2005) demonstrated that the changes in wavenumber encountered by mountain waves propagating through a translating barotropic jet were sufficient to change the magnitude of the horizontally averaged momentum flux. In future work, it would be interesting to use ray tracing to follow the mountain waves as they are modified while propagating through the complex large-scale flow to test whether such WKB effects are responsible for filling out the $k^{-5 / 3} \mathrm{KE}$ spectrum.

Acknowledgments. We greatly benefited from correspondence with Michael Waite and Jun Peng, discussions with Rich Rotunno, and comments from two anonymous reviewers. This research was funded by NSF Grant AGS-1545927. Author Menchaca was also supported by a National Science Foundation Graduate Research Fellowship. We would like to acknowledge high-performance computing support from Yellowstone (ark:/85065/d7wd3xhc) provided by NCAR's Computational and Information Systems Laboratory, sponsored by the National Science Foundation. 


\section{APPENDIX}

\section{Terms in the Spectral KE Budget}

Following Peng et al. (2015a), for two scalar fields $a$ and $b$ define $(a, b)_{\mathbf{k}}=\hat{a}^{*}(\mathbf{k}) \hat{b}(\mathbf{k})$, where $\hat{a}$ is the Fourier transform of $a$ and the asterisk denotes the complex conjugate. For the vector fields $\mathbf{a}$ and $\mathbf{b}$, define $(\mathbf{a}, \mathbf{b})_{\mathbf{k}}=\hat{\mathbf{a}}^{*}(\mathbf{k}) \cdot \hat{\mathbf{b}}(\mathbf{k})$. Using this notation and (1),

$$
\left.\partial_{t} E_{h}(\mathbf{k})=\frac{\bar{\rho}}{2}\left[\left(\mathbf{u}, \partial_{t} \mathbf{u}\right)_{\mathbf{k}}\right)+\text { c.c. }\right],
$$

where "c.c." denotes the complex conjugate of the preceding expression. Letting $\nabla$ be the $2 \mathrm{D}$ horizontal gradient operator, the horizontal momentum equation may be well approximated as

$$
\partial_{t} \mathbf{u}=-\mathbf{u} \cdot \nabla \mathbf{u}-w \partial_{z} \mathbf{u}-c_{p} \bar{\theta} \nabla \pi^{\prime}+\mathbf{F}_{h},
$$

where $\mathbf{u}$ is the horizontal velocity vector, $\mathbf{F}_{h}$ is the turbulent subgrid-scale diffusivity, $\bar{\theta}(z)$ is a vertically varying approximation to the full $\theta(x, y, z, t)$, and $\pi$ is the Exner function, which is split between a horizontally uniform component $\bar{\pi}(z)$ in hydrostatic balance with $\bar{\theta}(z)$ and the remainder $\pi^{\prime}(x, y, z, t)$. Substituting (A2) into (A1) gives

$$
\partial_{t} E_{h}(\mathbf{k})=A(\mathbf{k})+P(\mathbf{k})+D(\mathbf{k}) .
$$

Here $A$ is the tendency arising from advection,

$$
A(\mathbf{k})=-\frac{\bar{\rho}}{2}\left(\mathbf{u}, \mathbf{u} \cdot \nabla \mathbf{u}+w \partial_{z} \mathbf{u}\right)_{\mathbf{k}}+\text { c.c. },
$$

$P$ is from the pressure gradient force,

$$
P(\mathbf{k})=-\frac{\bar{\rho}}{2} c_{p} \bar{\theta}\left(\mathbf{u}, \nabla \pi^{\prime}\right)_{\mathbf{k}}+\text { c.c. }
$$

and $D$ is from dissipation,

$$
D(\mathbf{k})=\frac{\bar{\rho}}{2}\left(\mathbf{u}, \mathbf{F}_{h}\right)_{\mathbf{k}}+\text { c.c. }
$$

As detailed in Peng et al. (2014), Peng et al. (2015a), and Sun et al. (2017), the advection term can be decomposed as

$$
A(\mathbf{k})=T(\mathbf{k})+V_{a}(\mathbf{k})+\varepsilon_{1}(\mathbf{k}),
$$

where

$$
\begin{aligned}
T(\mathbf{k})= & -\bar{\rho}\left\{\left[\mathbf{u}, \mathbf{u} \cdot \nabla \mathbf{u}+\frac{1}{2} \mathbf{u}(\nabla \cdot \mathbf{u})\right]_{\mathbf{k}}-\frac{1}{2}\left(\partial_{z} \mathbf{u}, w \mathbf{u}\right)_{\mathbf{k}}\right. \\
& \left.+\frac{1}{2}\left(\mathbf{u}, w \partial_{z} \mathbf{u}\right)_{\mathbf{k}}\right\}+ \text { c.c., }
\end{aligned}
$$

represents that component of the transfer of energy between wavenumbers that is conservative in the sense that its sum over all $\mathbf{k}$ is zero. All derivatives are computed using a sixth-order compact difference [Lele 1992; Durran 2010, Eq. (3.50), p. 115]; horizontal derivatives are computed on constant $z$ surfaces. Our numerical formulas are, therefore, not algebraically identical to the corresponding terms in the WRF Model.

In (A7),

$$
V_{a}(\mathbf{k})=-\frac{1}{2} \partial_{z}\left[\bar{\rho}(\mathbf{u}, w \mathbf{u})_{\mathbf{k}}\right]+\text { c.c. },
$$

is the divergence of the vertical advective energy flux, and

$$
\varepsilon_{1}(\mathbf{k})=\frac{\bar{\rho}_{2}}{2}\left\{\left[\mathbf{u}, \mathbf{u}\left(\nabla \cdot \mathbf{u}+\partial_{z} w\right)\right]_{\mathbf{k}}+\partial_{z} \ln (\bar{\rho})(\mathbf{u}, w \mathbf{u})_{\mathbf{k}}\right\}+\text { c.c. }
$$

If the anelastic continuity equation, $\bar{\rho} \nabla \cdot \mathbf{u}+\partial_{z}(\bar{\rho} w)=0$, is satisfied, $\varepsilon_{1}(\mathbf{k})$ is zero. Although we are not using an anelastic model, $\varepsilon_{1}(\mathbf{k})$ is quite small in our simulations and will be neglected.

Peng et al. (2014) used the pseudo-incompressible approximation (Durran 1989) to write the contribution from the pressure gradient force as

$$
P(\mathbf{k})=c_{p} \bar{\rho} \bar{\theta}\left(w, \partial_{z} \pi^{\prime}\right)_{\mathbf{k}}-c_{p} \partial_{z}\left[\bar{\rho} \bar{\theta}\left(w, \pi^{\prime}\right)_{\mathbf{k}}\right]+\text { c.c. }
$$

Under the hydrostatic approximation, $\partial_{z} \pi^{\prime}$ is proportional to $\theta^{\prime}$ and the first term in (A11) represents buoyancy forcing, which will be subsequently written as

$$
B(\mathbf{k})=c_{p} \bar{\rho} \bar{\theta}\left(w, \partial_{z} \pi^{\prime}\right)_{\mathbf{k}}+\text { c.c. }
$$

The second term in (A11) is the divergence of the vertical flux of energy due to pressure working and will be combined with $V_{a}(\mathbf{k})$ to give the total vertical energy flux divergence

$$
V(\mathbf{k})=-\frac{1}{2} \partial_{z}\left[\bar{\rho}(\mathbf{u}, w \mathbf{u})_{\mathbf{k}}\right]-c_{p} \partial_{z}\left[\bar{\rho} \bar{\theta}\left(w, \pi^{\prime}\right)_{\mathbf{k}}\right]+\text { c.c. }
$$

The expression for $\mathbf{F}_{h}$ in the dissipation term, (A6), is evaluated as

$$
K_{h} \nabla^{2} \mathbf{u}+K_{v} \frac{\partial^{2} \mathbf{u}}{\partial z^{2}},
$$

where $K_{h}$ is the horizontal eddy viscosity computed by the WRF model from a function of the horizontal deformation and $K_{v}$ is vertical eddy viscosity computed 
from the boundary layer scheme. Because in most cases the dissipation is insignificant, we used the easy-tocompute approximate expression (A14) rather than the full expression for diffusion in physical space used in the actual WRF simulations. The correct full expression would compute the divergence of the deviatoric stress tensor and would move the eddy viscosities inside that divergence operator.

\section{REFERENCES}

Augier, P., and E. Lindborg, 2013: A new formulation of the spectral energy budget of the atmosphere, with application to two high-resolution general circulation models. J. Atmos. Sci., 70, 2293-2308, https://doi.org/10.1175/JAS-D-12-0281.1.

Bierdel, L., P. Friederichs, and S. Bentzien, 2012: Spatial kinetic energy spectra in the convection-permitting limited-area NWP model COSMO-DE. Meteor. Z., 21, 245-258, https:// doi.org/10.1127/0941-2948/2012/0319.

Burgess, B., A. Erler, and T. Shepherd, 2013: The troposphere-tostratosphere transition in kinetic energy spectra and nonlinear spectral fluxes as seen in ECMWF analyses. J. Atmos. Sci., 70, 669-687, https://doi.org/10.1175/JAS-D-12-0129.1.

Charney, J., 1971: Geostrophic turbulence. J. Atmos. Sci., 28, 1087-1095, https://doi.org/10.1175/1520-0469(1971)028<1087: GT>2.0.CO;2.

Chen, C.-C., D. R. Durran, and G. Hakim, 2005: Mountain-wave momentum flux in an evolving synoptic-scale flow. J. Atmos. Sci., 62, 3213-3231, https://doi.org/10.1175/JAS3543.1.

Dewan, E. M., 1979: Wave spectra resembling turbulence. Science, 204, 832-835, https://doi.org/10.1126/science.204.4395.832.

Durran, D. R., 1989: Improving the anelastic approximation. J. Atmos. Sci., 46, 1453-1461, https://doi.org/10.1175/1520-0469(1989) 046<1453:ITAA $>2.0$. CO;2.

—, 2010: Numerical Methods for Fluid Dynamics: With Applications to Geophysics. 2nd ed. Springer-Verlag, $516 \mathrm{pp}$.

—_, and J. Weyn, 2016: Thunderstorms don't get butterflies. Bull. Amer. Meteor. Soc., 97, 237-243, https://doi.org/10.1175/ BAMS-D-15-00070.1.

—_, and M. Menchaca, 2019: The influence of vertical wind shear on the evolution of mountain-wave momentum flux. J. Atmos Sci., 76, 749-756, https://doi.org/10.1175/JAS-D-18-0231.1.

_- J. Weyn, and M. Menchaca, 2017: Computing dimensional spectra from gridded data and compensating for discretization errors. Mon. Wea. Rev., 145, 3901-3910, https:// doi.org/10.1175/MWR-D-17-0056.1.

Eliassen, A., and E. Palm, 1960: On the transfer of energy in stationary mountain waves. Geofys. Publ., 22, 1-23.

Gage, K., 1979: Evidence for a $k^{-5 / 3}$ law inertial range in mesoscale two-dimensional turbulence. J. Atmos. Sci., 36, 1950-1954, https://doi.org/10.1175/1520-0469(1979)036<1950:EFALIR> 2.0.CO;2.

Garrett, C., and W. Munk, 1979: Internal waves in the ocean. Annu. Rev. Fluid Mech., 11, 339-369, https://doi.org/10.1146/ annurev.fl.11.010179.002011.

Hamilton, K., Y. Takahasi, and W. Ohfuchi, 2014: Mesoscale spectrum of atmospheric motions investigated in a very fine resolution global general circulation model. J. Geophys. Res., 113, D18110, https://doi.org/10.1029/2008JD009785.

Hong, S.-Y., Y. Noh, and J. Dudhia, 2006: A new vertical diffusion package with an explicit treatment of entrainment processes.
Mon. Wea. Rev., 134, 2318-2341, https://doi.org/10.1175/ MWR3199.1.

Jasperson, W., G. Nastrom, and D. Fritts, 1990: Further study of terrain effects on the mesoscale spectrum of atmospheric motions. J. Atmos. Sci., 47, 979-987, https://doi.org/10.1175/ 1520-0469(1990)047<0979:FSOTEO>2.0.CO;2.

Kim, Y.-H., H.-Y. Chun, S.-H. Park, I.-S. Song, and H.-J. Choi, 2016: Characteristics of gravity waves generated in the jetfront system in a baroclinic instability simulation. Atmos. Chem. Phys., 16, 4799-4815, https://doi.org/10.5194/acp-164799-2016.

Koshyk, J., and K. Hamilton, 2001: The horizontal kinetic energy spectrum and spectral budget simulated by a high-resolution troposphere-stratosphere-mesosphere GSM. J. Atmos. Sci., 58, 329-348, https://doi.org/10.1175/1520-0469(2001) $058<0329$ :THKESA $>2.0 . \mathrm{CO} ; 2$.

Kraichnan, R. H., 1967: Inertial ranges in two-dimensional turbulence. Phys. Fluids, 10, 1417-1423, https://doi.org/10.1063/ 1.1762301 .

Lele, S. K., 1992: Compact finite difference schemes with spectrallike resolution. J. Comput. Phys., 103, 16-42, https://doi.org/ 10.1016/0021-9991(92)90324-R.

Lilly, D., 1983: Stratified turbulence and the mesoscale variability of the atmosphere. J. Atmos. Sci., 40, 749-761, https://doi.org/ 10.1175/1520-0469(1983)040<0749:STATMV>2.0.CO;2.

Lindborg, E., 1999: Can the atmospheric kinetic energy spectrum be explained by two-dimensional turbulence? J. Fluid Mech., 388, 259-288, https://doi.org/10.1017/S0022112099004851.

Malardel, S., and N. Wedi, 2016: How does subgrid-scale parameterisation influence non-linear spectral energy fluxes in global NWP models? J. Geophys. Res. Atmos., 121, 5395-5410, https:// doi.org/10.1002/2015JD023970.

Menchaca, M. Q., and D. R. Durran, 2017: Mountain waves, downslope winds, and low-level blocking forced by a midlatitude cyclone encountering an isolated ridge. J. Atmos. Sci., 74, 617-639, https://doi.org/10.1175/JAS-D-16-0092.1.

$\longrightarrow$, and - 2018: The impact of mountain waves on an idealized baroclinically unstable large-scale flow. J. Atmos. Sci., 75, 3285-3302, https://doi.org/10.1175/JAS-D-17-0396.1.

Nastrom, G., and K. Gage, 1985: A climatology of atmospheric wavenumber spectra of wind and temperature observed by commercial aircraft. J. Atmos. Sci., 42, 950-960, https://doi.org/ 10.1175/1520-0469(1985)042<0950:ACOAWS > 2.0.CO;2.

— gravity waves. Part I: Topographic excitation. J. Atmos. Sci., 49, 101-110, https://doi.org/10.1175/1520-0469(1992)049<0101: SOMVOG $>2.0 . \mathrm{CO} ; 2$.

,-- , and K. Gage, 1987: An investigation of terrain effects on the mesoscale spectrum of atmospheric motions. J. Atmos. Sci., 44, 3087-3096, https://doi.org/10.1175/1520-0469(1987) 044<3087:AIOTEO > 2.0.CO;2.

Peng, J., L. Zhang, Y. Luo, and Y. Zhang, 2014: Mesoscale energy spectra of the Mei-Yu front system. Part I: Kinetic energy spectra. J. Atmos. Sci., 71, 37-55, https://doi.org/10.1175/JASD-13-085.1.

- - - , and J. Guan, 2015a: Applications of a moist nonhydrostatic formulation of the spectral energy budget to baroclinic waves. Part I: The lower-stratospheric energy spectra. J. Atmos. Sci., 72, 2090-2108, https://doi.org/10.1175/JAS-D14-0306.1.

- - , and,$- 2015 \mathrm{~b}$ : Applications of a moist nonhydrostatic formulation of the spectral energy budget to baroclinic waves. Part II: The upper-tropospheric energy spectra. 
J. Atmos. Sci., 72, 3923-3939, https://doi.org/10.1175/JAS-D14-0359.1.

Plougonven, R., and C. Snyder, 2007: Inertia-gravity waves spontaneously generated by jets and fronts. Part I: Different baroclinic life cycles. J. Atmos. Sci., 64, 2502-2519, https://doi.org/10.1175/JAS3953.1.

Selz, T., L. Bierdel, and G. C. Craig, 2019: Estimation of the variability of mesoscale energy spectra with three years of COSMO-DE analyses. J. Atmos. Sci., 76, 627-637, https://doi.org/10.1175/JAS-D-18-0155.1.

Skamarock, W., S.-H. Park, J. Klemp, and C. Snyder, 2014: Atmospheric kinetic energy spectra from global high-resolution nonhydrostatic simuations. J. Atmos. Sci., 71, 4369-4381, https://doi.org/10.1175/JAS-D-14-0114.1.

Smith, R. B., B. Woods, J. Jensen, W. Cooper, J. Doyle, Q. Jiang, and V. Grubisić, 2008: Mountain waves entering the stratosphere. J. Atmos. Sci., 65, 2543-2562, https://doi.org/10.1175/2007JAS2598.1.

- , and Coauthors, 2016: Stratospheric gravity wave fluxes and scales during DEEPWAVE. J. Atmos. Sci., 73, 2851-2869, https://doi.org/10.1175/JAS-D-15-0324.1.

Snyder, C., W. C. Skamarock, and R. Rotunno, 1993: Frontal dynamics near and following frontal collapse. J. Atmos. Sci., 50, 3194-3211, https://doi.org/10.1175/1520-0469(1993)050<3194: FDNAFF $>2.0 . \mathrm{CO} ; 2$.

Stumpf, M. P. H., and M. A. Porter, 2012: Critical truths about power laws. Science, 335, 665-666, https://doi.org/10.1126/ science.1216142.
Sun, Y. Q., R. Rotunno, and F. Zhang, 2017: Contributions of moist convection and internal gravity waves to building the atmospheric $-5 / 3$ kinetic energy spectra. J. Atmos. Sci., 74, 185-201, https://doi.org/10.1175/JAS-D-16-0097.1.

Vallis, G. K., 2017: Atmospheric and Oceanic Fluid Dynamics: Fundamentals and Large-Scale Circulation. 2nd ed. Cambridge University Press, 946 pp.

VanZandt, T., 1982: A universal spectrum of buoyancy waves in the atmosphere. Geophys. Res. Lett., 9, 575-578, https:// doi.org/10.1029/GL009i005p00575.

Waite, M., and C. Snyder, 2009: The mesoscale kinetic energy spectrum of a baroclinic life cycle. J. Atmos. Sci., 66, 883-901, https://doi.org/10.1175/2008JAS2829.1.

_ waves. J. Atmos. Sci., 70, 1242-1256, https://doi.org/10.1175/ JAS-D-11-0347.1.

Weyn, J., and D. Durran, 2017: The dependence of the predictability of mesoscale convective systems on the horizontal scale and amplitude of initial errors in idealized simulations. J. Atmos. Sci., 74, 2191-2210, https://doi.org/10.1175/JAS-D17-0006.1.

— , and - , 2019: The scale dependence of initial-condition sensitivities in simulations of convective systems over the southeastern United States. Quart. J. Roy. Meteor. Soc., https://doi.org/10.1002/qj.3367, in press. 